\title{
Imine Insertion into a Late Metal-Carbon Bond to Form a Stable Amido Complex
}

\author{
Christopher Krug and John F. Hartwig* \\ Department of Chemistry, Yale University, PO Box 208107, New Haven, CT 06520-8107
}

\section{Supporting Information}

\section{Table of Contents}

General Experimental Procedure and Reagent Availability $\quad$ S3

Preparation of (DPPE)Rh(pyridine)(p-tolyl) (2) $\quad$ S4

Preparation of (DPPE)Rh(pyridine) $\left(\mathrm{NArCH}(p-\text { tol })_{2}(4)\left(\mathrm{Ar}=\mathrm{C}_{6} \mathrm{H}_{4}-p-\mathrm{CO}_{2} \mathrm{Me}\right) \quad\right.$ S5

Reaction of 2 with $(\mathrm{Ph}) \mathrm{CH}=\mathrm{NPh}(\mathbf{3 b}) \quad$ S5

Independent Preparation of $(\mathrm{Ph})(p$-tol $) \mathrm{C}=\mathrm{NPh}(\mathbf{6}) \quad$ S6

Independent Preparation of $(\mathrm{Ph})(p$-tol)CHNHPh (5) S6

Representative Kinetic Experiment of 2 with $(\mathrm{Ph}) \mathrm{CH}=\mathrm{N}\left(\mathrm{C}_{6} \mathrm{H}_{4}-p-\mathrm{CO}_{2} \mathrm{Me}\right)(\mathbf{3 a}-\mathbf{P h}) \quad \mathrm{S} 7$

Figure S1. Plot of $\mathrm{k}_{\text {obs }}$ versus 1/[pyridine] $\quad$ S8

Reaction of $\mathbf{2}$ with $\mathbf{3 b}$ in the Presence of Added $(\mathrm{Ph})(p$-tol $) \mathrm{C}=\mathrm{N}\left(\mathrm{C}_{6} \mathrm{H}_{4}-p-\mathrm{CO}_{2} \mathrm{Me}\right)(7) \quad \mathrm{S} 8$

Independent Preparation of $(\mathrm{Ph})(p$-tol $) \mathrm{C}=\mathrm{N}\left(\mathrm{C}_{6} \mathrm{H}_{4}-p-\mathrm{CO}_{2} \mathrm{Me}\right)(7) \quad \mathrm{S} 8$

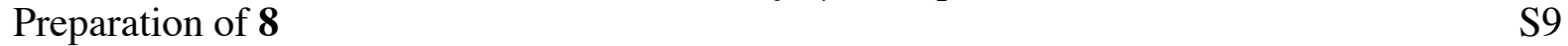

Deuterium Labeling Study in the Reaction of 2 and ( $p$-tol) $\mathrm{CH}=\mathrm{N}\left(\mathrm{C}_{6} \mathrm{H}_{4}-p\right.$-OMe) (3c) $\quad \mathrm{S} 10$

$\begin{array}{ll}\text { Thermolysis of } \mathbf{4} & \mathrm{S} 10\end{array}$

Independent Preparation of $(p \text {-tol })_{2} \mathrm{CHNH}\left(\mathrm{C}_{6} \mathrm{H}_{4}-p-\mathrm{CO}_{2} \mathrm{Me}\right)(\mathbf{9}) \quad \mathrm{S} 11$

Independent Preparation of $(p \text {-tol })_{2} \mathrm{C}=\mathrm{N}\left(\mathrm{C}_{6} \mathrm{H}_{4}-p-\mathrm{CO}_{2} \mathrm{Me}\right)(\mathbf{1 0}) \quad \mathrm{S} 11$

$\begin{array}{ll}\text { References } & \mathrm{S} 12\end{array}$

Experimental Procedure for the X-ray Diffraction of $2 \quad$ S13

Table S1. Crystal Data and Structure Refinement for $2 \quad$ S15

Table S2. Atomic Coordinates and Equivalent Isotopic Displacement Parameters for $2 \quad$ S16

Table S3. Bond Lengths and Bond Angles for $2 \quad$ S18

Table S4. Anisotropic Displacement Parameters for $2 \quad$ S20

Table S5. Hydrogen Coordinates and Isotopic Displacement Parameters for $2 \quad$ S22

$\begin{array}{ll}\text { Figure S2. ORTEP diagram of } 2 & \text { S24 }\end{array}$

$\begin{array}{ll}\text { Experimental Procedure for the X-ray Diffraction of } 4 & \text { S25 }\end{array}$

Table S6. Crystal Data and Structure Refinement for $4 \quad$ S27

Table S7. Atomic Coordinates and Equivalent Isotopic Displacement Parameters for 4 S28

Table S8. Bond Lengths and Bond Angles for $4 \quad$ S31

Table S9. Anisotropic Displacement Parameters for $4 \quad$ S34

Table S10. Hydrogen Coordinates and Isotopic Displacement Parameters for $4 \quad$ S37

$\begin{array}{ll}\text { Figure S3. ORTEP diagram of } 4 & \text { S39 }\end{array}$

Experimental Procedure for the X-ray Diffraction of $8 \quad$ S40 
Table S11. Crystal Data and Structure Refinement for 8

Table S12. Atomic Coordinates and Equivalent Isotopic Displacement Parameters for 8 S43

Table S13. Bond Lengths and Bond Angles for 8

Table S14. Anisotropic Displacement Parameters for 8

Table S15. Hydrogen Coordinates and Isotopic Displacement Parameters for $\mathbf{8}$

Figure S4. ORTEP diagram of 8

Figure S5. ${ }^{1} \mathrm{H}$ NMR spectrum of 8

S53

Figure S6. ${ }^{31} \mathrm{P}$ NMR spectrum of 8 
General. Unless noted otherwise, all manipulations were carried out under an inert atmosphere using a nitrogen-filled glovebox or standard Schlenk techniques. All glassware was oven-dried for approximately $1 \mathrm{~h}$ prior to use. THF, toluene, diethyl ether and pentane were distilled from sodium benzophenone ketyl under nitrogen. $\mathrm{C}_{6} \mathrm{D}_{6}$ and THF- $d_{8}$ were purchased from Cambridge Isotope Laboratories, Inc., dried over sodium benzophenone ketyl, and vacuum transferred prior to use. Column chromatography was performed using Merck silica gel 60 (230-400 mesh). ${ }^{1} \mathrm{H}$ NMR spectra were obtained on either a General Electric QE Plus 300-MHz spectrometer or Bruker DPX 400- or $500-\mathrm{MHz}$ spectrometers. ${ }^{1} \mathrm{H}$ NMR spectra were recorded relative to residual protiated solvent. ${ }^{13} \mathrm{C}$ NMR spectra were obtained at 100.6 or $125.0 \mathrm{MHz}$ on a Bruker DPX 400- or $500 \mathrm{MHz}$ instrument, and chemical shifts were recorded relative to the solvent resonance. Both ${ }^{1} \mathrm{H}$ NMR and ${ }^{13} \mathrm{C}$ NMR chemical shifts are reported in parts per million downfield from tetramethylsilane. ${ }^{31} \mathrm{P}$ NMR spectra were obtained at 121.6, 202.4 or 162.0 MHz on a General Electric Omega 300-MHz, $500-\mathrm{MHz}$ or a Bruker DPX 400-MHz instrument and chemical shifts are reported relative to $85 \%$ $\mathrm{H}_{3} \mathrm{PO}_{4}$. Kinetic experiments were performed on a General Electric Omega 500-MHz instrument. The temperature of the NMR probe was calibrated using a digital thermometer. Elemental analyses were performed by Robertson Microlit Laboratories, Inc., Madison, NJ or Atlantic Microlabs, Norcross, GA. [(DPPE)Rh( $\square-\mathrm{Cl})]_{2},{ }^{1}$ 4-methylbenzophenone imine, ${ }^{2}$ 4,4'-dimethylbenzophenone imine, ${ }^{2}(p-$ tol $) \mathrm{CH}=\mathrm{N}\left(\mathrm{C}_{6} \mathrm{H}_{4}-p-\mathrm{CO}_{2} \mathrm{Me}\right)^{3}$ (3a-Tol), $(\mathrm{Ph}) \mathrm{CH}=\mathrm{N}\left(\mathrm{C}_{6} \mathrm{H}_{4}-p-\mathrm{CO}_{2} \mathrm{Me}\right)^{3}$ (3a-Ph), $(\mathrm{Ph}) \mathrm{CH}=\mathrm{NPh}^{3}(\mathbf{3 b})$ and $(p$-tol $) \mathrm{CH}=\mathrm{N}\left(\mathrm{C}_{6} \mathrm{H}_{4}-p-\mathrm{OMe}\right)^{3}(\mathbf{3 c})$ were prepared using procedures reported for the preparation of similar compounds. $\mathrm{PhBr}$, NaO-t-Bu, $\mathrm{Cs}_{2} \mathrm{CO}_{3}, \mathrm{C}_{6} \mathrm{H}_{4} \mathrm{Br}-p-\mathrm{CO}_{2} \mathrm{Me}$, dodecahydrotriphenylene, $\mathrm{Et}_{3} \mathrm{NHCl}, \mathrm{DCl}\left(1.0 \mathrm{M}\right.$ solution in $\left.\mathrm{Et}_{2} \mathrm{O}\right)$, and $\mathrm{NaBH}_{4}$ were purchased from Aldrich and used as received. $\mathrm{Pd}_{2}(\mathrm{dba})_{3}$ and $( \pm$ )-BINAP were purchased from Strem and used as received. $\mathrm{Pd}(\mathrm{OAc})_{2}$ was purchased from Next Chimica. Pyridine was purchased from Fisher 
Chemical and was degassed by sparging with nitrogen prior to use. Anhydrous EtOH was purchased from Pharmco and used as received. Solid $p$-tolyllithium was prepared according to a literature procedure. $^{4}$

Preparation of (dppe)Rh(pyridine)(p-tolyl) (2). Into a $50 \mathrm{~mL}$ round bottom flask equipped with a magnetic stir bar was placed $\left[(\mathrm{DPPE}) \mathrm{Rh}(\square-\mathrm{Cl})_{2}\right](185 \mathrm{mg}, 0.172 \mathrm{mmol})$. THF $(10 \mathrm{~mL})$ and pyridine (209 $\square \mathrm{L}, 2.58 \mathrm{mmol})$ were added to generate a cloudy yellow solution after ca. $10 \mathrm{~min} . \mathrm{p}$ Tolyllithium (34.6 mg, $0.353 \mathrm{mmol}$ ) was added as a solid, and an orange solution was generated. After 5 min of stirring at room temperature, all volatile materials were removed under reduced pressure. The product was extracted into $\mathrm{Et}_{2} \mathrm{O}$, and the resulting solution filtered through Celite. The clear orange solution was slowly concentrated and cooled at $-35^{\circ} \mathrm{C}$ to give $150.0 \mathrm{mg}$ of orange crystalline 2 (65\% yield). ${ }^{1} \mathrm{H}$ NMR (400 MHz, $\left.\mathrm{C}_{6} \mathrm{D}_{6}\right): \square 1.87-2.05(\mathrm{~m}, 4 \mathrm{H}), 2.20(\mathrm{~s}, 3 \mathrm{H}), 6.08(\mathrm{t}$, $J=6.8 \mathrm{~Hz}, 2 \mathrm{H}), 6.46(\mathrm{t}, J=8.0 \mathrm{~Hz}, 1 \mathrm{H}), 6.91(\mathrm{~d}, J=6.8 \mathrm{~Hz}, 2 \mathrm{H}), 7.05-7.16(\mathrm{~m}, 12 \mathrm{H}), 7.64-7.73(\mathrm{~m}$, $6 \mathrm{H})$, 7.83-7.88 (m, 4H), 8.53-8.55 (m, 2H). ${ }^{13} \mathrm{C}$ NMR (125.8 MHz, THF- $\left.d_{8}\right): \square 21.3,28.5-28.8(\mathrm{~m})$, 31.0-31.5 (m), 124.2, 126.6 (d, J=5.7 Hz), 127.9 (d, J=9.3 Hz), 128.5, 128.6 (d, J=8.4 Hz), 129.0, 129.7, 133.8 (d, J=12.3 Hz), 134.4 (d, J=11.6 Hz), 135.7, 138.7 (d, J=38.2 Hz), 139.3 (d, J=21.1 $\mathrm{Hz}), 139.4,153.2,175.0\left(\mathrm{ddd}, J_{\mathrm{RhC}}=94.1 \mathrm{~Hz},{ }^{2} J_{\mathrm{CP}}=31.5 \mathrm{~Hz},{ }^{2} J_{\mathrm{CP}}=15.0 \mathrm{~Hz}\right) .{ }^{31} \mathrm{P} \mathrm{NMR}(202 \mathrm{MHz}$, $\left.\mathrm{C}_{6} \mathrm{D}_{6}\right): \square 57.6\left(\mathrm{dd}, J_{\mathrm{RhP}}=122 \mathrm{~Hz}, J_{\mathrm{PP}}=19.6 \mathrm{~Hz}\right), 74.3\left(\mathrm{dd}, J_{\mathrm{RhP}}=200 \mathrm{~Hz}, J_{\mathrm{PP}}=19.6 \mathrm{~Hz}\right)$. Anal. Calcd for $\mathrm{C}_{38} \mathrm{H}_{36} \mathrm{NP}_{2} \mathrm{Rh}: \mathrm{C}, 67.96 ; \mathrm{H}, 5.40 ; \mathrm{N}, 2.09$. Found: C, 67.69; H, 5.30; N, 1.94.

Preparation of 4 . Into a $20 \mathrm{~mL}$ scintillation vial equipped with stir bar was placed 2 (112 $\mathrm{mg}, 0.167 \mathrm{mmol})$ and 3a-Tol (46.4 mg, $0.184 \mathrm{mmol}, 1.1$ equiv). Toluene (6-8 mL) was added, and the solution stirred for 10-15 min at room temperature. The solution was concentrated under reduced pressure to $1 \mathrm{~mL}$ and cooled at $-35^{\circ} \mathrm{C}$ for $12 \mathrm{~h}$. An orange solid precipitated, which was isolated from the supernatant and washed with cold toluene and dried to give $150 \mathrm{mg}$ of $4(88 \%)$. 
Complex 4 was stored at $-35{ }^{\circ} \mathrm{C} .{ }^{1} \mathrm{H}$ NMR (400 MHz, THF- $\left.d_{8},-20{ }^{\circ} \mathrm{C}\right): \square 1.54-2.25(\mathrm{~m}, 4 \mathrm{H}), 2.10$ (s, 3H), $2.31(\mathrm{~s}, 3 \mathrm{H}), 2.37(\mathrm{~s}, 3 \mathrm{H}), 3.57(\mathrm{~s}, 3 \mathrm{H}), 4.84(\mathrm{~s}, 1 \mathrm{H}), 5.49$ (dd, J=9.4 Hz, J=2.2 Hz, 1H), $6.32(\mathrm{~d}$, $J=7.6 \mathrm{~Hz}, 2 \mathrm{H}), 6.46(\mathrm{~d}, J=7.6 \mathrm{~Hz}, 2 \mathrm{H}), 6.59$ (d, $J=4 \mathrm{~Hz}, 2 \mathrm{H}), 6.78$ (d, J=8.0 Hz, 2H), 7.10-7.50 (m, $26 \mathrm{H}), 7.69(\mathrm{~m}, 4 \mathrm{H}), 8.45(\mathrm{~d}, J=5.2 \mathrm{~Hz}, 2 \mathrm{H}), 8.51(\mathrm{dd}, J=9.0 \mathrm{~Hz}, J=2.2 \mathrm{~Hz}, 1 \mathrm{H}) .{ }^{31} \mathrm{P}$ NMR (162 MHz, THF- $\left.d_{8},-20{ }^{\circ} \mathrm{C}\right): \square 67.5(\mathrm{~d}, J=176.8 \mathrm{~Hz})$. Anal. Calcd for $\mathrm{C}_{61} \mathrm{H}_{59} \mathrm{~N}_{2} \mathrm{O}_{2} \mathrm{P}_{2} \mathrm{Rh}: \mathrm{C}, 72.04 ; \mathrm{H}, 5.85$; N, 2.75. Found: C, 72.04; H, 5.90; N, 2.52.

Reaction of 2 with PhCH=NPh (3b). To a small vial was placed 2 (10.0 mg, 0.0149 mmol) and dodecahydrotriphenylene $\left(1 \mathrm{mg}\right.$ ) as internal standard. These compounds were dissolved in $\mathrm{C}_{6} \mathrm{D}_{6}$ (0.7 mL), and an ${ }^{1} \mathrm{H}$ NMR spectrum was acquired. The solution was then added to a separate vial containing 3b (13.6 mg, $0.0744 \mathrm{mmol}, 5$ equiv), and the resulting solution stirred at room temperature for $1 \mathrm{~h} . \mathrm{Et}_{3} \mathrm{NHCl}(6.20 \mathrm{mg}, 0.0447 \mathrm{mmol}, 3$ equiv) was added, and the solution stirred for $2 \mathrm{~h}$ or until the solution had changed from a dark color to an orange color. An ${ }^{1} \mathrm{H}$ NMR spectrum was acquired, and yields of ketimine $\mathbf{6}$ and diarylmethylamine 5 were calculated to be 50 and $25 \%$.

Independent Preparation of $\boldsymbol{N}$-Phenyl-4-methylbenzophenone Imine (6). ${ }^{5}$ Into a $20 \mathrm{~mL}$ scintillation vial was placed 4-methylbenzophenone imine (500 mg, $2.56 \mathrm{mmol})$, bromobenzene (402 mg, $2.56 \mathrm{mmol}), \mathrm{Pd}_{2}(\mathrm{dba})_{3}(6.60 \mathrm{mg}, 0.00641 \mathrm{mmol}, 0.25 \mathrm{~mol} \%)$, ( \pm )-BINAP (12.0 mg, 0.0192 mmol, $0.75 \mathrm{~mol} \%)$ and $\mathrm{NaOt}$-Bu (345 mg, $3.59 \mathrm{mmol}, 1.4$ equiv). Toluene (10 mL) was added, and the vial capped. The mixture was heated at $80{ }^{\circ} \mathrm{C}$ for $12 \mathrm{~h}$. The yellow solution was allowed to cool to room temperature and was filtered through Celite. The volatile materials were removed under reduced pressure, and the imine was purified by silica gel chromatography eluting with $10 \%$ ethyl acetate in hexane. Compound $\mathbf{6}(636 \mathrm{mg}, 92 \%)$ was obtained as a 1:1 mixture of $E$ and $Z$ isomers. ${ }^{1} \mathrm{H}$ NMR (300 MHz, $\left.\mathrm{C}_{6} \mathrm{D}_{6}\right): \square 1.91(\mathrm{~s}, 3 \mathrm{H}), 2.05(\mathrm{~s}, 3 \mathrm{H}), 6.72(\mathrm{~d}, J=7.8 \mathrm{~Hz}, 2 \mathrm{H}), 6.74-7.06(\mathrm{~m}, 22 \mathrm{H})$, $7.94(\mathrm{~d}, J=8.1 \mathrm{~Hz}, 2 \mathrm{H}), 7.98-8.01(\mathrm{~m}, 2 \mathrm{H})$. 
Independent Preparation of $N$-Phenyl(phenyl $p$-tolylmethyl)amine (5). ${ }^{6}$ The following reaction was not conducted in an inert atmosphere. Into a $250 \mathrm{~mL}$ round bottom flask equipped with a magnetic stir bar was placed ketimine 6 (200 mg, $0.738 \mathrm{mmol})$. The ketimine was dissolved in EtOH (30 mL), and $\mathrm{NaBH}_{4}(2.21 \mathrm{~g}, 59.0 \mathrm{mmol}, 80$ equiv) was added in four portions. The yellow color eventually dissipated to give a colorless solution after 2-3 h. When the reaction was complete, as determined by TLC, the volatile materials were removed by rotary evaporation. The remaining solid was quenched with water and the product was extracted into $\mathrm{Et}_{2} \mathrm{O}$. The organic layer was separated and dried over $\mathrm{MgSO}_{4}$ and filtered. The $\mathrm{Et}_{2} \mathrm{O}$ was removed by rotary evaporation and the residue was purified by silica gel chromatography eluting with $30 \%$ ethyl acetate in hexane to give $173 \mathrm{mg}(86 \%)$ of the diarylmethylamine. ${ }^{1} \mathrm{H}$ NMR $\left(300 \mathrm{MHz}, \mathrm{C}_{6} \mathrm{D}_{6}\right)$ : $\square 2.08(\mathrm{~s}, 3 \mathrm{H}), 3.91(\mathrm{~d}, \mathrm{~J}=3.6$ Hz, 1H), 5.41 (d, J=4.2 Hz, 1H), $6.44(\mathrm{~d}, J=7.8 \mathrm{~Hz}, 2 \mathrm{H}), 6.70(\mathrm{~m}, 1 \mathrm{H}), 6.93$ (d, J=7.8 Hz, 2H), 7.01$7.25(\mathrm{~m}, 9 \mathrm{H})$.

\section{Representative Procedure for the Kinetic Experiments of the reaction between 2 and}

3a-Ph. Into a small vial was placed $2(12.0 \mathrm{mg}, 0.0179 \mathrm{mmol})$, toluene $(0.40 \mathrm{~mL})$, and pyridine $(5.1$ $\square \mathrm{L}, 0.0625 \mathrm{mmol}, 3.5$ equiv). This solution was transferred to an NMR tube equipped with a screwcapped top containing a Teflon septum. The solution was cooled with dry ice. In a separate vial was placed imine 3a-Ph (21.5 mg, $0.0893 \mathrm{mmol}, 5$ equiv), which was then dissolved in toluene $(0.30$ $\mathrm{mL}$ ). This solution was drawn into a $1 \mathrm{~mL}$ syringe and added to the cold solution contained in the NMR tube. The contents of the NMR tube were mixed briefly, and the tube was placed into the NMR probe cooled to $2.0{ }^{\circ} \mathrm{C}$. The consumption of 2 was monitored by ${ }^{31} \mathrm{P}$ NMR spectroscopy, and the rate constants were determined by fitting the data to an exponential decay using KaleidaGraph software. This procedure was repeated using 5, 8, 11 and 18 equiv of pyridine. 


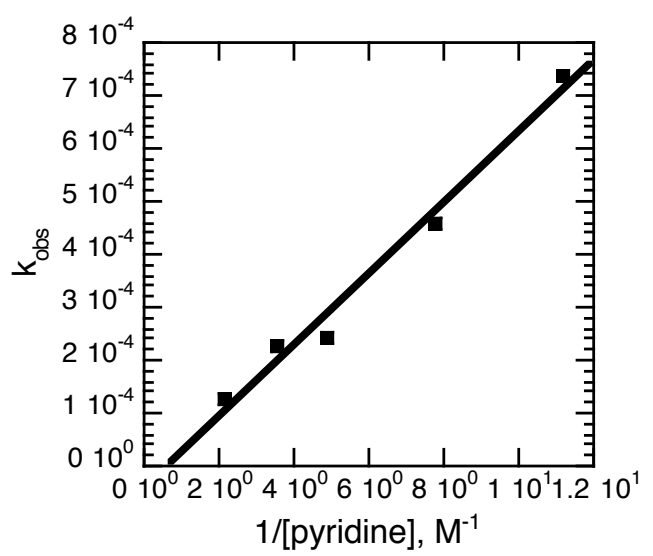

Figure S1. Plot of $\mathrm{k}_{\mathrm{obs}}$ versus $1 /[$ pyridine].

Reaction of 2 with $\mathrm{PhCH}=\mathrm{NPh}(3 \mathrm{~b})$ in the Presence of Added Ketimine 7. To a small vial was placed 2 (10.0 mg, $0.0149 \mathrm{mmol}), 3 \mathbf{b}(3.00 \mathrm{mg}, 0.0164 \mathrm{mmol}, 1.1$ equiv) and 7 (4.90 mg, $0.0149 \mathrm{mmol})$. These compounds were dissolved in $\mathrm{C}_{6} \mathrm{D}_{6}(0.7 \mathrm{~mL})$, and the resulting solution was stirred for 2 h. GC-MS analysis of the solution showed the formation of amine $\mathbf{5}$ and ketimine $\mathbf{6}$ and no formation of the diarylmethylamine from 7.

Independent Preparation of Ketimine 7. To a $20 \mathrm{~mL}$ scintillation vial equipped with magnetic stir bar was placed 4-methylbenzophenone imine (0.500 g, $2.56 \mathrm{mmol}), \mathrm{C}_{6} \mathrm{H}_{4} \mathrm{Br}-p-\mathrm{CO}_{2} \mathrm{Me}$ (543 mg, $2.56 \mathrm{mmol}), \mathrm{Pd}(\mathrm{OAc})_{2}(11.5 \mathrm{mg}, 0.0513 \mathrm{mmol}, 2 \mathrm{~mol} \%),( \pm)-\mathrm{BINAP}$ (47.9 mg, 0.0769 mmol, $3 \mathrm{~mol} \%)$ and $\mathrm{Cs}_{2} \mathrm{CO}_{3}(1.17 \mathrm{~g}, 3.59 \mathrm{mmol}, 1.4$ equiv). Toluene ( $\sim 8 \mathrm{~mL})$ was added, and the mixture heated to $100{ }^{\circ} \mathrm{C}$ for $5 \mathrm{~h}$. The yellow solution was allowed to cool to room temperature and filtered. The volatile materials were removed under reduced pressure, and the ketimine was purified by silica gel chromatography using $10 \%$ ethyl acetate in hexane as eluent. $689 \mathrm{mg}(82 \%)$ of the ketimine was obtained as a 1:1 mixture of $E$ and $Z$ isomers. ${ }^{1} \mathrm{H}$ NMR $\left(400 \mathrm{MHz}, \mathrm{C}_{6} \mathrm{D}_{6}\right): \square 1.90$ (s, 3H), $2.05(\mathrm{~s}, 3 \mathrm{H}), 3.42(\mathrm{~s}, 3 \mathrm{H}), 3.43(\mathrm{~s}, 3 \mathrm{H}), 6.70-6.74(\mathrm{~m}, 6 \mathrm{H}), 6.83(\mathrm{~d}, J=7.6 \mathrm{~Hz}, 2 \mathrm{H}), 6.86-6.91$ $(\mathrm{m}, 6 \mathrm{H}), 6.98(\mathrm{~d}, J=8.0 \mathrm{~Hz}, 2 \mathrm{H}), 7.88(\mathrm{~d}, J=8.0 \mathrm{~Hz}, 2 \mathrm{H}), 7.93-7.95(\mathrm{~m}, 2 \mathrm{H}), 7.99-8.01(\mathrm{~m}, 6 \mathrm{H}) .{ }^{13} \mathrm{C}$ NMR (100 MHz, $\left.\mathrm{CDCl}_{3}\right)$ : $\square 21.3,21.4,51.8,120.5,120.6,124.5,127.9,128.2,128.7,128.8,129.0$, 
129.3, 129.4, 129.5, 130.2, 130.3, 131.0, 132.5, 135.7, 136.2, 139.2, 141.6, 155.7, 155.8, 166.9,

168.7, 169.0. Anal. Calcd for $\mathrm{C}_{22} \mathrm{H}_{19} \mathrm{NO}_{2}: \mathrm{C}, 80.22 ; \mathrm{H}, 5.81 ; \mathrm{N}, 4.25$. Found: $\mathrm{C}, 79.98 ; \mathrm{H}, 5.90 ; \mathrm{N}$, 4.02 .

Preparation of Cyclometalated Complex 8. To a $20 \mathrm{~mL}$ scintillation vial equipped with a magnetic stir bar was placed $2(114 \mathrm{mg}, 0.170 \mathrm{mmol})$ and imine $3 \mathbf{c}(57.3 \mathrm{mg}, 0.255 \mathrm{mmol}, 1.5$ equiv). Toluene (4-6 mL) was added, and the resulting solution was stirred at room temperature for $3 \mathrm{~h}$. The dark solution was concentrated in vacuo and was layered with pentane and cooled overnight at $-35{ }^{\circ} \mathrm{C}$. An olive green solid precipitated. The dark supernatant was removed with a pipette, and the solid was washed with pentane and dried in vacuo to give $62.0 \mathrm{mg}$ of $\mathbf{8}$ (44\%). Dark green crystals were obtained for elemental analysis by recrystallization of $\mathbf{8}$ from cold toluene/pentane. Crystals suitable for X-ray diffraction were grown by vapor diffusion of pentane into a concentrated toluene solution of 8. ${ }^{1} \mathrm{H}$ NMR $\left(400 \mathrm{MHz}, \mathrm{C}_{6} \mathrm{D}_{6}\right)$ : $\square 1.77(\mathrm{~s}, 3 \mathrm{H}), 1.85(\mathrm{~m}, 4 \mathrm{H})$, $1.95(\mathrm{~s}, 3 \mathrm{H}), 2.94(\mathrm{~s}, 3 \mathrm{H}), 5.93(\mathrm{~d}, J=7.6 \mathrm{~Hz}, 2 \mathrm{H}), 6.60-6.62(\mathrm{~m}, 4 \mathrm{H}), 6.68(\mathrm{~d}, J=7.6 \mathrm{~Hz}, 1 \mathrm{H}), 6.84$ (d, $J=8.0 \mathrm{~Hz}, 2 \mathrm{H}), 6.98-7.16(\mathrm{~m}, 13 \mathrm{H}), 7.51$ (br s, $1 \mathrm{H}), 7.73-7.77(\mathrm{~m}, 4 \mathrm{H}), 8.32-8.36(\mathrm{~m}, 4 \mathrm{H}) .{ }^{31} \mathrm{P}$ NMR (121.6 MHz, $\left.\mathrm{C}_{6} \mathrm{D}_{6}\right): \square 55.0\left(\mathrm{dd}, J_{\mathrm{PRh}}=123 \mathrm{~Hz}, J_{\mathrm{PP}}=19.5 \mathrm{~Hz}\right), 76.3\left(\mathrm{dd}, J_{\mathrm{PRh}}=203 \mathrm{~Hz}, J_{\mathrm{PP}}=19.5\right.$ $\mathrm{Hz})$.

\section{Deuterium Labeling Study of the Reaction of 2 with $(p$-tol $) \mathrm{CH}=\mathrm{N}\left(\mathrm{C}_{6} \mathrm{H}_{4}-p\right.$-OMe $)(3 \mathrm{c})$.}

To a small vial was placed $2(10.0 \mathrm{mg}, 0.0149 \mathrm{mmol})$ and $\mathbf{3 c}(3.70 \mathrm{mg}, 0.0164 \mathrm{mmol}, 1.1$ equiv). These compounds were dissolved in $\mathrm{C}_{6} \mathrm{D}_{6}(0.7 \mathrm{~mL})$, and the resulting solution was stirred for $3 \mathrm{~h}$. $\mathrm{DCl}$ (48.0 $\square \mathrm{L}$ of a $1.0 \mathrm{M} \mathrm{Et}_{2} \mathrm{O}$ solution, $0.0447 \mathrm{mmol}, 3$ equiv) was added by syringe. After $15 \mathrm{~min}$, 10 drops of a $1 \mathrm{~N}$ aqueous $\mathrm{HCl}$ solution were added, and the contents were stirred overnight at room temperature. A $6 \mathrm{M}$ aqueous solution of $\mathrm{NaOH}$ was added dropwise until the solution was basic. GC-MS analysis of the $\mathrm{C}_{6} \mathrm{D}_{6}$ layer showed the products of ketimine hydrolysis (4,4'- 
dimethylbenzophenone and $p$-anisidine). $(\mathrm{M}+1) /(\mathrm{M}+)$ Ratios (authentic material in parentheses): $p$ anisidine, 0.083 (0.073); 4,4'-dimethylbenzophenone, 2.39 (0.14).

Thermolysis of 4 . To a small vial was placed 2 (10.0 $\mathrm{mg}, 0.0149 \mathrm{mmol})$ and 1,3,5trimethoxybenzene $(1 \mathrm{mg})$ as internal standard. These compounds were dissolved in $\mathrm{C}_{6} \mathrm{D}_{6}(0.7$ $\mathrm{mL}$ ), and a ${ }^{1} \mathrm{H}$ NMR spectrum was acquired. The solution was then added to a separate vial containing 3a-Tol (3.56 mg, $0.0156 \mathrm{mmol}, 1.05$ equiv), and the resulting solution was stirred at room temperature for $10 \mathrm{~min}$. The red-orange solution was transferred to an NMR tube and heated to $75{ }^{\circ} \mathrm{C}$ for about $0.5 \mathrm{~h} . \mathrm{Et}_{3} \mathrm{NHCl}(6.20 \mathrm{mg}, 0.0447 \mathrm{mmol}, 3$ equiv) was added, and the solution was stirred for $2 \mathrm{~h}$ or until the it had changed from a dark color to a red-orange color. A ${ }^{1} \mathrm{H}$ NMR spectrum was acquired, and the yields of diarylmethylamine $\mathbf{9}$ and ketimine $\mathbf{1 0}$ were calculated to be 50 and $45 \%$.

Independent Preparation of 9. This reaction was not conducted under an inert atmosphere. Into a $250 \mathrm{~mL}$ round bottom flask equipped with a magnetic stir bar was placed ketimine 10 (95.0 mg, $0.277 \mathrm{mmol})$. The ketimine was suspended in $\mathrm{MeOH}(30 \mathrm{~mL})$, and $\mathrm{NaBH}_{4}(0.842 \mathrm{~g}, 22.2 \mathrm{mmol}, 80$ equiv $)$ was added in four portions. The yellow color of the ketimine faded after 2-3 h of heating at reflux. However, the ketimine was not completely consumed, as determined by TLC, after additional heating at reflux or upon addition of additional $\mathrm{NaBH}_{4}$. The volatile materials were removed by rotary evaporation, and the remaining solid was quenched with water and the product was extracted into $\mathrm{Et}_{2} \mathrm{O}$. The organic layer was separated and dried over $\mathrm{MgSO}_{4}$ and filtered. The $\mathrm{Et}_{2} \mathrm{O}$ was removed by rotary evaporation, and the residue was purified by silica gel chromatography eluting with $10 \%$ ethyl acetate in hexane to give $51.1 \mathrm{mg}(54 \%)$ of 9. ${ }^{1} \mathrm{H}$ NMR $\left(500 \mathrm{MHz}, \mathrm{C}_{6} \mathrm{D}_{6}\right)$ : $\square 2.08(\mathrm{~s}, 6 \mathrm{H}), 3.52$ (s, 3H), 4.28 (d, J=5.0 Hz, 1H), 5.37 (d, J=5.0 Hz, 1H), 6.28 (d, J=7.3 Hz, $2 \mathrm{H}), 6.94$ (d, J=8.0 
$\mathrm{Hz}, 4 \mathrm{H}), 7.06(\mathrm{~d}, J=8.5 \mathrm{~Hz}, 4 \mathrm{H}), 8.07(\mathrm{~d}, J=7.0 \mathrm{~Hz}, 2 \mathrm{H}) .{ }^{13} \mathrm{C} \mathrm{NMR}\left(125.8 \mathrm{MHz}, \mathrm{C}_{6} \mathrm{D}_{6}\right): \square 21.0$, 51.1, 61.9, 112.9, 119.7, 127.9, 129.6, 131.7, 137.2, 139.7, 151.2, 166.9. Anal. Calcd for $\mathrm{C}_{23} \mathrm{C}_{23} \mathrm{NO}_{2}: \mathrm{C}, 79.97 ; \mathrm{H}, 6.71 ; \mathrm{N}, 4.05$. Found:C, 79.75; H, 6.95; N, 3.91.

Independent Preparation of 10. To a $20 \mathrm{~mL}$ scintillation vial equipped with a magnetic stir bar was placed 4,4'-dimethylbenzophenone imine (0.500 g, $2.39 \mathrm{mmol}), \mathrm{C}_{6} \mathrm{H}_{4} \mathrm{Br}-p-\mathrm{CO}_{2} \mathrm{Me}$ (540 mg, $2.63 \mathrm{mmol}, 1.10$ equiv), $\mathrm{Pd}(\mathrm{OAc})_{2}$ (10.7 mg, $\left.0.0478 \mathrm{mmol}, 2 \mathrm{~mol} \%\right),( \pm)$-BINAP (44.7 $\mathrm{mg}, 0.0718 \mathrm{mmol}, 3 \mathrm{~mol} \%)$ and $\mathrm{Cs}_{2} \mathrm{CO}_{3}(1.09 \mathrm{~g}, 3.35 \mathrm{mmol}, 1.4$ equiv). Toluene ( $8 \mathrm{~mL})$ was added, and the mixture was heated at $100{ }^{\circ} \mathrm{C}$ for $5 \mathrm{~h}$. The yellow solution was allowed to cool to room temperature and filtered. The volatile materials were removed under reduced pressure, and the ketimine was purified by silica gel chromatography using $10 \%$ ethyl acetate in hexane as eluent. Recrystallization from EtOH gave $410 \mathrm{mg}(50 \%)$ of $\mathbf{1 0} .{ }^{1} \mathrm{H}$ NMR $\left(400 \mathrm{MHz}, \mathrm{C}_{6} \mathrm{D}_{6}\right)$ : $1.91(\mathrm{~s}, 3 \mathrm{H}), 2.07$ (s, 3H), $3.42(\mathrm{~s}, 3 \mathrm{H}), 6.74(\mathrm{~m}, 4 \mathrm{H}), 6.88$ (d, J=7.6 Hz, 2H), 7.00 (d, J=8.0 Hz, 2H), $7.92(\mathrm{~d}, J=8.4 \mathrm{~Hz}, 2 \mathrm{H}), 7.99(\mathrm{~d}, J=8.4 \mathrm{~Hz}, 2 \mathrm{H}) .{ }^{13} \mathrm{C} \mathrm{NMR}\left(100 \mathrm{MHz}, \mathrm{C}_{6} \mathrm{D}_{6}\right): \square 21.1,21.3$, $51.2,120.9,125.2,128.9,129.2,129.6,130.0,130.8,133.4,137.2,138.7,141.4,156.7,166.4$, 168.3. Anal. Calcd for $\mathrm{C}_{23} \mathrm{C}_{21} \mathrm{NO}_{2}: \mathrm{C}, 80.44 ; \mathrm{H}, 6.16 ; \mathrm{N}, 4.08$. Found: $\mathrm{C}, 80.46 ; \mathrm{H}, 5.71 ; \mathrm{N}$, 3.67.

\section{References}

1) Fairlie, D. P.; Bosnich, B. Organometallics 1988, 7, 936.

2) Cristau, H. -J.; Lambert, J. -M.; Pirat, J. -L. Synthesis 1998, 1167.

3) Castellano, J. A.; Goldmacher, J. E.; Barton, L. A.; Kane, J. S. J. Org. Chem. 1968, 33, 3501.

4) Chetcuti, M. J.; Chisholm, M. H.; Folting, C. K.; Haitko, D. A.; Huffman, J. C.; Janos, J. J. Am. Chem. Soc. 1983, 105, 1163. 
5) Arndtsen, B. A.; Sleiman, H. F.; Chang, A. K.; McElwee-White, L. J. Am. Chem. Soc. 1991, $113,4871$.

6) Zhu, Z.; Espenson, J. H. J. Org. Chem. 1996, 61, 324.

\section{Experimental Procedure for the X-ray Diffraction of 2}

\section{$\underline{\text { Data Collection }}$}

An orange plate crystal of $\mathrm{C}_{38} \mathrm{H}_{36} \mathrm{NP}_{2} \mathrm{Rh}$ having approximate dimensions of $0.15 \mathrm{x} 0.10 \mathrm{x}$ $0.08 \mathrm{~mm}^{3}$ was mounted with epoxy cement on the tip of a fine glass fiber. All measurements were made on a Bruker Platform APEX-CCD diffractometer with graphite monochromated Mo$\mathrm{K} \square$ radiation.

Cell constants and an orientation matrix for data collection corresponded to a triclinic cell with dimensions:

$$
\begin{array}{ll}
\mathrm{a}=11.1280(18) \AA & \square=84.261(3)^{\mathrm{o}} \\
\mathrm{b}=11.1646(18) \AA & \square=84.977(3)^{\mathrm{o}} \\
\mathrm{c}=13.098(2) \AA & \square=80.620(3)^{\mathrm{o}} \\
\mathrm{V}=1593.3(4) \AA^{3} &
\end{array}
$$

For $Z=2$ and F.W. $=671.53$, the calculated density is $1.400 \mathrm{~g} / \mathrm{cm}^{3}$. Based on a statistical analysis of intensity distribution, and the successful solution and refinement of the structure, the space group was determined to be $P-1$ (\#2).

The data were collected at a temperature of $150(2) \mathrm{K}$ to a maximum $2 \square$ value of $56.52^{\circ}$. Four omega/phi scans consisting 2400 data frames, respectively, were collected with a frame width of $0.3^{\circ}$ and a detector-to-crystal distance, Dx, of $49 \mathrm{~mm}$. The data frames were processed and scaled using the Bruker-SAINT software package. ${ }^{1}$

\section{Data Reduction}

A total of 18538 reflections were collected of which 7376 were unique and observed $\left(R_{\text {int }}\right.$ $=0.0420$ ). The linear absorption coefficient, $\square$, for Mo-K $\square$ radiation is $6.64 \mathrm{~cm}^{-1}$. An empirical absorption correction was applied and the data were corrected for Lorentz and polarization effects.

\section{$\underline{\text { Structure Solution and Refinement }}$}

The structure was solved by direct methods and expanded using Fourier techniques ${ }^{2}$. The non-hydrogen atoms were refined anisotropically, and hydrogen atoms were treated as idealized 
contributions. The final cycle of full-matrix least-squares refinement ${ }^{3}$ on $\mathrm{F}$ was based on 7376 observed reflections (I $>2.00 \square(\mathrm{I})$ ) and 379 variable parameters and converged with unweighted and weighted agreement factors of:

$$
\begin{gathered}
\mathrm{R}=\square\|\mathrm{Fo}|-| \mathrm{Fc}\| / \square|\mathrm{Fo}|=0.0435 \\
\mathrm{R}_{\mathrm{W}}=\left\{\square\left[\mathrm{w}\left(\mathrm{F}_{\mathrm{o}}{ }^{2}-\mathrm{F}_{\mathrm{c}}{ }^{2}\right)^{2}\right] / \square\left[\mathrm{w}\left(\mathrm{F}_{\mathrm{o}}{ }^{2}\right)^{2}\right]\right\}^{1 / 2}=0.1012
\end{gathered}
$$

The maximum and minimum peaks on the final difference Fourier map corresponded to 1.059 and $-0.477 \mathrm{e}^{-} / \AA^{3}$ respectively.

\section{REFERENCES}

(1) Bruker-AXS, Inc., Madison, WI, 1997

(2) Acta Cryst. A46 (1990) 467-473

(3) Least Squares function minimized:

$$
\square w\left(\mathrm{~F}_{\mathrm{o}}^{2}-\mathrm{F}_{\mathrm{c}}{ }^{2}\right)^{2}
$$


Table S1. Crystal data and structure refinement for $\mathbf{2}$.

Empirical formula

Formula weight

Temperature

Wavelength

Crystal system

Space group

Unit cell dimensions

Volume

Z

Density (calculated)

Absorption coefficient

$\mathrm{F}(000)$

Crystal size

Theta range for data collection

Index ranges

Reflections collected

Independent reflections

Completeness to theta $=28.26^{\circ}$

Absorption correction

Refinement method

Data / restraints / parameters

Goodness-of-fit on F2

Final $\mathrm{R}$ indices [I $>2 \operatorname{sigma}(\mathrm{I})]$

$\mathrm{R}$ indices (all data)

Largest diff. peak and hole
$\mathrm{C}_{38} \mathrm{H}_{36} \mathrm{~N} \mathrm{P}_{2} \mathrm{Rh}$

671.53

150(2) K

$0.71073 \AA$

Triclinic

P-1

$\mathrm{a}=11.1280(18) \AA$

$\mathrm{a}=84.261(3)^{\circ}$.

$\mathrm{b}=11.1646(18) \AA$

$\mathrm{b}=84.977(3)^{\circ}$.

$\mathrm{c}=13.098(2) \AA$

1593.3(4) А̊3

2

$1.400 \mathrm{~g} / \mathrm{cm} 3$

$6.64 \mathrm{~cm}-1$

692

$0.15 \times 0.10 \times 0.08 \mathrm{~mm} 3$

1.86 to $28.26^{\circ}$.

$-14<=\mathrm{h}<=14,-14<=\mathrm{k}<=14,-17<=\mathrm{l}<=17$

18538

$7376[\mathrm{R}(\mathrm{int})=0.0420]$

$93.6 \%$

\section{SADABS}

Full-matrix least-squares on F2

7376 / 0 / 379

1.085

$\mathrm{R} 1=0.0435, \mathrm{wR} 2=0.1012$

$\mathrm{R} 1=0.0570, \mathrm{wR} 2=0.1066$

1.268 and -0.747 e. $\AA \AA-3$ 
Table S2. Atomic coordinates ( x 104) and equivalent isotropic displacement parameters ( $\AA 2$ x 103 ) for 2 . $U(e q)$ is defined as one third of the trace of the orthogonalized Uij tensor.

\begin{tabular}{|c|c|c|c|c|}
\hline & $\mathrm{x}$ & $\mathrm{y}$ & $\mathrm{z}$ & $\mathrm{U}(\mathrm{eq})$ \\
\hline $\mathrm{Rh}(1)$ & $7428(1)$ & $5897(1)$ & $6843(1)$ & $21(1)$ \\
\hline $\mathrm{P}(1)$ & $7287(1)$ & $6607(1)$ & $8347(1)$ & $23(1)$ \\
\hline $\mathrm{P}(2)$ & $7406(1)$ & $4068(1)$ & $7729(1)$ & $22(1)$ \\
\hline $\mathrm{N}(1)$ & $7514(2)$ & $5261(2)$ & $5353(2)$ & $27(1)$ \\
\hline$C(1)$ & $5822(3)$ & $7515(3)$ & $8749(2)$ & $25(1)$ \\
\hline$C(2)$ & 4926(3) & $7809(3)$ & $8047(3)$ & $31(1)$ \\
\hline$C(3)$ & $3809(3)$ & $8502(3)$ & $8323(3)$ & $39(1)$ \\
\hline$C(4)$ & $3592(3)$ & $8906(3)$ & $9298(3)$ & $38(1)$ \\
\hline$C(5)$ & 4471(3) & $8612(3)$ & 9999(3) & $35(1)$ \\
\hline$C(6)$ & $5579(3)$ & 7918(3) & $9728(2)$ & $30(1)$ \\
\hline$C(7)$ & $8408(3)$ & $7541(3)$ & $8622(2)$ & $24(1)$ \\
\hline $\mathrm{C}(8)$ & 9634(3) & $7002(3)$ & $8628(2)$ & $30(1)$ \\
\hline$C(9)$ & $10545(3)$ & $7689(3)$ & $8710(3)$ & $35(1)$ \\
\hline$C(10)$ & $10256(3)$ & $8930(3)$ & $8775(3)$ & $38(1)$ \\
\hline $\mathrm{C}(11)$ & $9052(3)$ & $9488(3)$ & $8762(3)$ & $35(1)$ \\
\hline$C(12)$ & $8127(3)$ & $8797(3)$ & $8688(2)$ & $29(1)$ \\
\hline$C(13)$ & 7483(3) & $5371(3)$ & $9404(2)$ & $28(1)$ \\
\hline$C(14)$ & 6962(3) & $4276(3)$ & $9103(2)$ & $26(1)$ \\
\hline$C(15)$ & 8918(3) & $3121(2)$ & $7725(2)$ & $24(1)$ \\
\hline$C(16)$ & $9590(3)$ & $2848(3)$ & $8579(3)$ & $32(1)$ \\
\hline$C(17)$ & 10752(3) & $2186(3)$ & $8513(3)$ & $39(1)$ \\
\hline$C(18)$ & $11249(3)$ & 1778(3) & $7586(3)$ & $39(1)$ \\
\hline$C(19)$ & $10604(3)$ & $2045(3)$ & $6717(3)$ & $37(1)$ \\
\hline$C(20)$ & $9449(3)$ & 2732(3) & $6777(3)$ & $31(1)$ \\
\hline$C(21)$ & $6400(3)$ & 2997(3) & $7490(2)$ & $24(1)$ \\
\hline$C(22)$ & 6784(3) & 1779(3) & $7309(2)$ & $28(1)$ \\
\hline$C(23)$ & $5947(3)$ & 1033(3) & $7149(2)$ & $33(1)$ \\
\hline$C(24)$ & $4718(3)$ & $1495(3)$ & $7168(3)$ & $39(1)$ \\
\hline$C(25)$ & $4322(3)$ & 2703(3) & $7326(3)$ & $40(1)$ \\
\hline$C(26)$ & $5155(3)$ & $3448(3)$ & $7487(3)$ & $34(1)$ \\
\hline $\mathrm{C}(27)$ & 7559(3) & 7631(3) & $6106(2)$ & $24(1)$ \\
\hline
\end{tabular}




$\begin{array}{lllll}\mathrm{C}(28) & 8537(3) & 8250(3) & 6184(2) & 28(1) \\ \mathrm{C}(29) & 8612(3) & 9403(3) & 5683(2) & 31(1) \\ \mathrm{C}(30) & 7714(3) & 9987(3) & 5063(2) & 33(1) \\ \mathrm{C}(31) & 6743(3) & 9377(3) & 4958(3) & 33(1) \\ \mathrm{C}(32) & 6678(3) & 8231(3) & 5474(2) & 30(1) \\ \mathrm{C}(33) & 7772(4) & 11234(3) & 4519(3) & 54(1) \\ \mathrm{C}(34) & 6650(3) & 4684(3) & 5052(2) & 31(1) \\ \mathrm{C}(35) & 6707(3) & 4254(3) & 4094(3) & 38(1) \\ \mathrm{C}(36) & 7704(4) & 4381(3) & 3421(3) & 46(1) \\ \mathrm{C}(37) & 8602(4) & 4959(3) & 3713(3) & 48(1) \\ \mathrm{C}(38) & 8452(3) & 5409(3) & 4672(3) & 35(1) \\ \end{array}$


Table S3. Bond lengths $[\AA]$ and angles $\left[{ }^{\circ}\right]$ for 2 .

\begin{tabular}{|c|c|c|c|}
\hline $\operatorname{Rh}(1)-C(27)$ & $2.097(3)$ & $\mathrm{C}(23)-\mathrm{C}(24)$ & $1.379(5)$ \\
\hline $\mathrm{Rh}(1)-\mathrm{N}(1)$ & $2.132(3)$ & $C(24)-C(25)$ & $1.378(5)$ \\
\hline $\mathrm{Rh}(1)-\mathrm{P}(1)$ & $2.1797(8)$ & $C(25)-C(26)$ & $1.384(4)$ \\
\hline $\mathrm{Rh}(1)-\mathrm{P}(2)$ & $2.2481(8)$ & $\mathrm{C}(27)-\mathrm{C}(32)$ & $1.382(4)$ \\
\hline $\mathrm{P}(1)-\mathrm{C}(7)$ & $1.830(3)$ & $\mathrm{C}(27)-\mathrm{C}(28)$ & $1.397(4)$ \\
\hline $\mathrm{P}(1)-\mathrm{C}(1)$ & $1.838(3)$ & $\mathrm{C}(28)-\mathrm{C}(29)$ & $1.397(4)$ \\
\hline $\mathrm{P}(1)-\mathrm{C}(13)$ & $1.855(3)$ & $\mathrm{C}(29)-\mathrm{C}(30)$ & $1.381(4)$ \\
\hline $\mathrm{P}(2)-\mathrm{C}(21)$ & $1.831(3)$ & $\mathrm{C}(30)-\mathrm{C}(31)$ & $1.390(5)$ \\
\hline $\mathrm{P}(2)-\mathrm{C}(15)$ & $1.835(3)$ & $\mathrm{C}(30)-\mathrm{C}(33)$ & $1.508(4)$ \\
\hline $\mathrm{P}(2)-\mathrm{C}(14)$ & $1.852(3)$ & $\mathrm{C}(31)-\mathrm{C}(32)$ & $1.396(4)$ \\
\hline $\mathrm{N}(1)-\mathrm{C}(38)$ & $1.334(4)$ & $\mathrm{C}(34)-\mathrm{C}(35)$ & $1.380(4)$ \\
\hline $\mathrm{N}(1)-\mathrm{C}(34)$ & $1.346(4)$ & $\mathrm{C}(35)-\mathrm{C}(36)$ & $1.372(5)$ \\
\hline $\mathrm{C}(1)-\mathrm{C}(6)$ & $1.391(4)$ & $\mathrm{C}(36)-\mathrm{C}(37)$ & $1.374(5)$ \\
\hline $\mathrm{C}(1)-\mathrm{C}(2)$ & $1.393(4)$ & $\mathrm{C}(37)-\mathrm{C}(38)$ & $1.385(5)$ \\
\hline $\mathrm{C}(2)-\mathrm{C}(3)$ & $1.392(4)$ & & \\
\hline $\mathrm{C}(3)-\mathrm{C}(4)$ & $1.385(5)$ & $\mathrm{C}(27)-\mathrm{Rh}(1)-\mathrm{N}(1)$ & $87.50(10)$ \\
\hline $\mathrm{C}(4)-\mathrm{C}(5)$ & $1.377(5)$ & $\mathrm{C}(27)-\mathrm{Rh}(1)-\mathrm{P}(1)$ & $90.96(8)$ \\
\hline$C(5)-C(6)$ & $1.383(4)$ & $\mathrm{N}(1)-\mathrm{Rh}(1)-\mathrm{P}(1)$ & $177.77(7)$ \\
\hline$C(7)-C(12)$ & $1.396(4)$ & $\mathrm{C}(27)-\mathrm{Rh}(1)-\mathrm{P}(2)$ & 174.94(8) \\
\hline $\mathrm{C}(7)-\mathrm{C}(8)$ & $1.399(4)$ & $\mathrm{N}(1)-\mathrm{Rh}(1)-\mathrm{P}(2)$ & $96.16(7)$ \\
\hline $\mathrm{C}(8)-\mathrm{C}(9)$ & $1.384(4)$ & $\mathrm{P}(1)-\mathrm{Rh}(1)-\mathrm{P}(2)$ & $85.47(3)$ \\
\hline$C(9)-C(10)$ & $1.380(5)$ & $\mathrm{C}(7)-\mathrm{P}(1)-\mathrm{C}(1)$ & $103.08(13)$ \\
\hline$C(10)-C(11)$ & $1.383(5)$ & $\mathrm{C}(7)-\mathrm{P}(1)-\mathrm{C}(13)$ & $101.22(14)$ \\
\hline$C(11)-C(12)$ & $1.398(4)$ & $\mathrm{C}(1)-\mathrm{P}(1)-\mathrm{C}(13)$ & $102.58(14)$ \\
\hline$C(13)-C(14)$ & $1.533(4)$ & $\mathrm{C}(7)-\mathrm{P}(1)-\mathrm{Rh}(1)$ & $118.75(10)$ \\
\hline$C(15)-C(16)$ & $1.382(4)$ & $\mathrm{C}(1)-\mathrm{P}(1)-\mathrm{Rh}(1)$ & $116.88(10)$ \\
\hline$C(15)-C(20)$ & $1.405(4)$ & $\mathrm{C}(13)-\mathrm{P}(1)-\mathrm{Rh}(1)$ & $112.03(10)$ \\
\hline$C(16)-C(17)$ & $1.381(5)$ & $\mathrm{C}(21)-\mathrm{P}(2)-\mathrm{C}(15)$ & $103.14(13)$ \\
\hline$C(17)-C(18)$ & $1.377(5)$ & $\mathrm{C}(21)-\mathrm{P}(2)-\mathrm{C}(14)$ & $100.55(13)$ \\
\hline$C(18)-C(19)$ & $1.380(5)$ & $\mathrm{C}(15)-\mathrm{P}(2)-\mathrm{C}(14)$ & 104.71(14) \\
\hline$C(19)-C(20)$ & $1.385(4)$ & $\mathrm{C}(21)-\mathrm{P}(2)-\mathrm{Rh}(1)$ & $123.90(10)$ \\
\hline$C(21)-C(22)$ & $1.394(4)$ & $\mathrm{C}(15)-\mathrm{P}(2)-\mathrm{Rh}(1)$ & $112.90(9)$ \\
\hline$C(21)-C(26)$ & $1.396(4)$ & $\mathrm{C}(14)-\mathrm{P}(2)-\mathrm{Rh}(1)$ & $109.55(10)$ \\
\hline$C(22)-C(23)$ & $1.388(4)$ & $\mathrm{C}(38)-\mathrm{N}(1)-\mathrm{C}(34)$ & $116.6(3)$ \\
\hline
\end{tabular}




\begin{tabular}{|c|c|c|c|}
\hline $\mathrm{C}(38)-\mathrm{N}(1)-\mathrm{Rh}(1)$ & $120.7(2)$ & $\mathrm{C}(32)-\mathrm{C}(27)-\mathrm{C}(28)$ & $115.1(3)$ \\
\hline $\mathrm{C}(34)-\mathrm{N}(1)-\mathrm{Rh}(1)$ & $122.7(2)$ & $\mathrm{C}(32)-\mathrm{C}(27)-\mathrm{Rh}(1)$ & $121.3(2)$ \\
\hline$C(6)-C(1)-C(2)$ & $119.0(3)$ & $\mathrm{C}(28)-\mathrm{C}(27)-\mathrm{Rh}(1)$ & $123.6(2)$ \\
\hline$C(6)-C(1)-P(1)$ & $122.3(2)$ & $C(27)-C(28)-C(29)$ & $122.8(3)$ \\
\hline $\mathrm{C}(2)-\mathrm{C}(1)-\mathrm{P}(1)$ & $118.8(2)$ & $\mathrm{C}(30)-\mathrm{C}(29)-\mathrm{C}(28)$ & $120.9(3)$ \\
\hline $\mathrm{C}(3)-\mathrm{C}(2)-\mathrm{C}(1)$ & $120.3(3)$ & $C(29)-C(30)-C(31)$ & $117.3(3)$ \\
\hline$C(4)-C(3)-C(2)$ & $119.8(3)$ & $\mathrm{C}(29)-\mathrm{C}(30)-\mathrm{C}(33)$ & $121.9(3)$ \\
\hline$C(5)-C(4)-C(3)$ & $120.3(3)$ & $\mathrm{C}(31)-\mathrm{C}(30)-\mathrm{C}(33)$ & $120.9(3)$ \\
\hline$C(4)-C(5)-C(6)$ & $120.0(3)$ & $\mathrm{C}(30)-\mathrm{C}(31)-\mathrm{C}(32)$ & $120.9(3)$ \\
\hline$C(5)-C(6)-C(1)$ & $120.7(3)$ & $\mathrm{C}(27)-\mathrm{C}(32)-\mathrm{C}(31)$ & $123.0(3)$ \\
\hline $\mathrm{C}(12)-\mathrm{C}(7)-\mathrm{C}(8)$ & $118.1(3)$ & $\mathrm{N}(1)-\mathrm{C}(34)-\mathrm{C}(35)$ & $123.1(3)$ \\
\hline $\mathrm{C}(12)-\mathrm{C}(7)-\mathrm{P}(1)$ & $123.1(2)$ & $\mathrm{C}(36)-\mathrm{C}(35)-\mathrm{C}(34)$ & 119.1(3) \\
\hline $\mathrm{C}(8)-\mathrm{C}(7)-\mathrm{P}(1)$ & $118.2(2)$ & $\mathrm{C}(35)-\mathrm{C}(36)-\mathrm{C}(37)$ & $118.9(3)$ \\
\hline $\mathrm{C}(9)-\mathrm{C}(8)-\mathrm{C}(7)$ & $121.2(3)$ & $\mathrm{C}(36)-\mathrm{C}(37)-\mathrm{C}(38)$ & $118.5(3)$ \\
\hline $\mathrm{C}(10)-\mathrm{C}(9)-\mathrm{C}(8)$ & $120.2(3)$ & $\mathrm{N}(1)-\mathrm{C}(38)-\mathrm{C}(37)$ & $123.7(3)$ \\
\hline $\mathrm{C}(9)-\mathrm{C}(10)-\mathrm{C}(11)$ & $119.9(3)$ & & \\
\hline$C(10)-C(11)-C(12)$ & $120.2(3)$ & & \\
\hline $\mathrm{C}(7)-\mathrm{C}(12)-\mathrm{C}(11)$ & $120.5(3)$ & & \\
\hline $\mathrm{C}(14)-\mathrm{C}(13)-\mathrm{P}(1)$ & $108.7(2)$ & & \\
\hline $\mathrm{C}(13)-\mathrm{C}(14)-\mathrm{P}(2)$ & $109.2(2)$ & & \\
\hline$C(16)-C(15)-C(20)$ & $118.7(3)$ & & \\
\hline $\mathrm{C}(16)-\mathrm{C}(15)-\mathrm{P}(2)$ & $123.7(2)$ & & \\
\hline $\mathrm{C}(20)-\mathrm{C}(15)-\mathrm{P}(2)$ & $117.4(2)$ & & \\
\hline$C(17)-C(16)-C(15)$ & $120.9(3)$ & & \\
\hline $\mathrm{C}(18)-\mathrm{C}(17)-\mathrm{C}(16)$ & $119.9(3)$ & & \\
\hline $\mathrm{C}(17)-\mathrm{C}(18)-\mathrm{C}(19)$ & $120.5(3)$ & & \\
\hline $\mathrm{C}(18)-\mathrm{C}(19)-\mathrm{C}(20)$ & $119.8(3)$ & & \\
\hline$C(19)-C(20)-C(15)$ & $120.2(3)$ & & \\
\hline $\mathrm{C}(22)-\mathrm{C}(21)-\mathrm{C}(26)$ & $118.1(3)$ & & \\
\hline $\mathrm{C}(22)-\mathrm{C}(21)-\mathrm{P}(2)$ & $125.1(2)$ & & \\
\hline $\mathrm{C}(26)-\mathrm{C}(21)-\mathrm{P}(2)$ & $116.8(2)$ & & \\
\hline $\mathrm{C}(23)-\mathrm{C}(22)-\mathrm{C}(21)$ & $120.9(3)$ & & \\
\hline $\mathrm{C}(24)-\mathrm{C}(23)-\mathrm{C}(22)$ & $119.9(3)$ & & \\
\hline $\mathrm{C}(25)-\mathrm{C}(24)-\mathrm{C}(23)$ & $120.1(3)$ & & \\
\hline$C(24)-C(25)-C(26)$ & $120.0(3)$ & & \\
\hline$C(25)-C(26)-C(21)$ & $120.9(3)$ & & \\
\hline
\end{tabular}


Table S4. Anisotropic displacement parameters $(\AA 2 \times 103)$ for $\mathbf{2}$. The anisotropic displacement factor exponent takes the form: $-2 \mathrm{p} 2\left[\mathrm{~h} 2 \mathrm{a} * 2 \mathrm{U} 11+\ldots+2 \mathrm{~h} \mathrm{k} \mathrm{a}^{*} \mathrm{~b} * \mathrm{U} 12\right]$

\begin{tabular}{|c|c|c|c|c|c|c|}
\hline & U11 & U22 & U33 & $\mathrm{U} 23$ & U13 & U12 \\
\hline $\operatorname{Rh}(1)$ & $24(1)$ & $16(1)$ & $24(1)$ & $-2(1)$ & $-2(1)$ & $-6(1)$ \\
\hline $\mathrm{P}(1)$ & $27(1)$ & $18(1)$ & $26(1)$ & $-3(1)$ & $-3(1)$ & $-6(1)$ \\
\hline $\mathrm{P}(2)$ & $25(1)$ & $18(1)$ & $26(1)$ & $-3(1)$ & $-2(1)$ & $-7(1)$ \\
\hline $\mathrm{N}(1)$ & $29(1)$ & $22(1)$ & $29(1)$ & $-3(1)$ & $-2(1)$ & $-3(1)$ \\
\hline $\mathrm{C}(1)$ & $26(2)$ & $17(1)$ & $34(2)$ & $-5(1)$ & $1(1)$ & $-8(1)$ \\
\hline$C(2)$ & $30(2)$ & $32(2)$ & $32(2)$ & $-9(1)$ & $-2(1)$ & $-9(1)$ \\
\hline$C(3)$ & $29(2)$ & $40(2)$ & $49(2)$ & $-7(2)$ & $-8(2)$ & $-4(2)$ \\
\hline$C(4)$ & $27(2)$ & $35(2)$ & $51(2)$ & $-12(2)$ & $7(2)$ & $-7(1)$ \\
\hline$C(5)$ & $36(2)$ & $34(2)$ & $37(2)$ & $-11(2)$ & $9(2)$ & $-12(2)$ \\
\hline$C(6)$ & $30(2)$ & $29(2)$ & $33(2)$ & $-5(1)$ & $1(1)$ & $-10(1)$ \\
\hline$C(7)$ & $29(2)$ & $21(1)$ & $23(2)$ & $-4(1)$ & $-2(1)$ & $-8(1)$ \\
\hline$C(8)$ & $32(2)$ & $28(2)$ & $31(2)$ & $-4(1)$ & $-5(1)$ & $-4(1)$ \\
\hline$C(9)$ & $25(2)$ & $44(2)$ & $38(2)$ & $-4(2)$ & $-5(1)$ & $-8(2)$ \\
\hline$C(10)$ & $36(2)$ & $41(2)$ & $42(2)$ & $-5(2)$ & $-6(2)$ & $-21(2)$ \\
\hline $\mathrm{C}(11)$ & $42(2)$ & $24(2)$ & $42(2)$ & $-2(1)$ & $-8(2)$ & $-14(1)$ \\
\hline$C(12)$ & $28(2)$ & $24(2)$ & $35(2)$ & $-2(1)$ & $-4(1)$ & $-6(1)$ \\
\hline$C(13)$ & $40(2)$ & $20(1)$ & $24(2)$ & $-2(1)$ & $-1(1)$ & $-8(1)$ \\
\hline $\mathrm{C}(14)$ & $34(2)$ & $20(1)$ & $25(2)$ & $-1(1)$ & $2(1)$ & $-8(1)$ \\
\hline$C(15)$ & $24(2)$ & $17(1)$ & $33(2)$ & $-4(1)$ & $-1(1)$ & $-9(1)$ \\
\hline$C(16)$ & $36(2)$ & $25(2)$ & $36(2)$ & $-4(1)$ & $-6(1)$ & $-7(1)$ \\
\hline$C(17)$ & $36(2)$ & $34(2)$ & $50(2)$ & $1(2)$ & $-15(2)$ & $-6(2)$ \\
\hline $\mathrm{C}(18)$ & $25(2)$ & $26(2)$ & $66(3)$ & $0(2)$ & $-3(2)$ & $-5(1)$ \\
\hline$C(19)$ & $33(2)$ & $28(2)$ & $49(2)$ & $-9(2)$ & $10(2)$ & $-6(1)$ \\
\hline$C(20)$ & $32(2)$ & $30(2)$ & $33(2)$ & $-8(1)$ & $2(1)$ & $-9(1)$ \\
\hline $\mathrm{C}(21)$ & $28(2)$ & $21(1)$ & $25(2)$ & $1(1)$ & $-5(1)$ & $-9(1)$ \\
\hline$C(22)$ & $29(2)$ & $25(2)$ & $32(2)$ & $-9(1)$ & $0(1)$ & $-7(1)$ \\
\hline$C(23)$ & $39(2)$ & $28(2)$ & $36(2)$ & $-9(1)$ & 1(1) & $-12(1)$ \\
\hline $\mathrm{C}(24)$ & $36(2)$ & $38(2)$ & $49(2)$ & $-7(2)$ & $-7(2)$ & $-20(2)$ \\
\hline$C(25)$ & $26(2)$ & $40(2)$ & $56(2)$ & $0(2)$ & $-11(2)$ & $-9(2)$ \\
\hline$C(26)$ & $31(2)$ & $24(2)$ & $46(2)$ & $-1(1)$ & $-6(2)$ & $-6(1)$ \\
\hline $\mathrm{C}(27)$ & $30(2)$ & $22(1)$ & $21(2)$ & $-4(1)$ & 2(1) & $-7(1)$ \\
\hline
\end{tabular}




\begin{tabular}{lllllll}
$\mathrm{C}(28)$ & $32(2)$ & $24(2)$ & $28(2)$ & $0(1)$ & $-2(1)$ & $-7(1)$ \\
$\mathrm{C}(29)$ & $38(2)$ & $26(2)$ & $31(2)$ & $-4(1)$ & $1(1)$ & $-14(1)$ \\
$\mathrm{C}(30)$ & $46(2)$ & $22(2)$ & $29(2)$ & $-2(1)$ & $-1(1)$ & $-6(1)$ \\
$\mathrm{C}(31)$ & $39(2)$ & $24(2)$ & $35(2)$ & $-3(1)$ & $-9(1)$ & $3(1)$ \\
$\mathrm{C}(32)$ & $30(2)$ & $30(2)$ & $32(2)$ & $-7(1)$ & $1(1)$ & $-9(1)$ \\
$\mathrm{C}(33)$ & $78(3)$ & $30(2)$ & $56(3)$ & $9(2)$ & $-10(2)$ & $-14(2)$ \\
$\mathrm{C}(34)$ & $35(2)$ & $26(2)$ & $33(2)$ & $-5(1)$ & $-6(1)$ & $-6(1)$ \\
$\mathrm{C}(35)$ & $49(2)$ & $27(2)$ & $38(2)$ & $-6(1)$ & $-14(2)$ & $-5(2)$ \\
$\mathrm{C}(36)$ & $73(3)$ & $35(2)$ & $31(2)$ & $-13(2)$ & $1(2)$ & $-9(2)$ \\
$\mathrm{C}(37)$ & $60(3)$ & $45(2)$ & $39(2)$ & $-11(2)$ & $13(2)$ & $-14(2)$ \\
$\mathrm{C}(38)$ & $39(2)$ & $31(2)$ & $36(2)$ & $-6(1)$ & $2(2)$ & $-11(2)$ \\
& & & & & & \\
\hline
\end{tabular}


Table S5. Hydrogen coordinates ( x 104) and isotropic displacement parameters ( $\AA 2$ x 103 ) for 2 .

\begin{tabular}{|c|c|c|c|c|}
\hline & $\mathrm{x}$ & $\mathrm{y}$ & z & $\mathrm{U}(\mathrm{eq})$ \\
\hline $\mathrm{H}(2 \mathrm{~A})$ & 5077 & 7536 & 7377 & 37 \\
\hline $\mathrm{H}(3 \mathrm{~A})$ & 3197 & 8696 & 7845 & 47 \\
\hline $\mathrm{H}(4 \mathrm{~A})$ & 2835 & 9387 & 9484 & 45 \\
\hline $\mathrm{H}(5 \mathrm{~A})$ & 4316 & 8887 & 10668 & 42 \\
\hline $\mathrm{H}(6 \mathrm{~A})$ & 6180 & 7714 & 10215 & 36 \\
\hline $\mathrm{H}(8 \mathrm{~A})$ & 9845 & 6150 & 8576 & 36 \\
\hline $\mathrm{H}(9 \mathrm{~A})$ & 11372 & 7304 & 8721 & 42 \\
\hline $\mathrm{H}(10 \mathrm{~A})$ & 10883 & 9400 & 8829 & 46 \\
\hline $\mathrm{H}(11 \mathrm{~A})$ & 8853 & 10343 & 8804 & 42 \\
\hline $\mathrm{H}(12 \mathrm{~A})$ & 7302 & 9185 & 8683 & 35 \\
\hline $\mathrm{H}(13 \mathrm{~A})$ & 8360 & 5132 & 9520 & 33 \\
\hline $\mathrm{H}(13 \mathrm{~B})$ & 7048 & 5657 & 10048 & 33 \\
\hline $\mathrm{H}(14 \mathrm{~A})$ & 6061 & 4418 & 9219 & 32 \\
\hline $\mathrm{H}(14 \mathrm{~B})$ & 7283 & 3533 & 9534 & 32 \\
\hline $\mathrm{H}(16 \mathrm{~A})$ & 9248 & 3120 & 9222 & 38 \\
\hline $\mathrm{H}(17 \mathrm{~A})$ & 11207 & 2011 & 9105 & 47 \\
\hline $\mathrm{H}(18 \mathrm{~A})$ & 12043 & 1310 & 7545 & 47 \\
\hline $\mathrm{H}(19 \mathrm{~A})$ & 10951 & 1759 & 6081 & 44 \\
\hline $\mathrm{H}(20 \mathrm{~A})$ & 9015 & 2941 & 6175 & 37 \\
\hline $\mathrm{H}(22 \mathrm{~A})$ & 7629 & 1456 & 7296 & 33 \\
\hline $\mathrm{H}(23 \mathrm{~A})$ & 6220 & 205 & 7027 & 40 \\
\hline $\mathrm{H}(24 \mathrm{~A})$ & 4144 & 981 & 7072 & 47 \\
\hline $\mathrm{H}(25 \mathrm{~A})$ & 3477 & 3023 & 7324 & 48 \\
\hline $\mathrm{H}(26 \mathrm{~A})$ & 4874 & 4278 & 7597 & 40 \\
\hline $\mathrm{H}(28 \mathrm{~A})$ & 9178 & 7870 & 6596 & 33 \\
\hline $\mathrm{H}(29 \mathrm{~A})$ & 9291 & 9791 & 5769 & 37 \\
\hline $\mathrm{H}(31 \mathrm{~A})$ & 6115 & 9746 & 4528 & 40 \\
\hline $\mathrm{H}(32 \mathrm{~A})$ & 5998 & 7845 & 5387 & 36 \\
\hline $\mathrm{H}(33 \mathrm{~A})$ & 8516 & 11518 & 4680 & 82 \\
\hline $\mathrm{H}(33 \mathrm{~B})$ & 7056 & 11803 & 4748 & 82 \\
\hline
\end{tabular}




\begin{tabular}{lrrrr}
$\mathrm{H}(33 \mathrm{C})$ & 7783 & 11191 & 3774 & 82 \\
$\mathrm{H}(34 \mathrm{~A})$ & 5969 & 4568 & 5520 & 37 \\
$\mathrm{H}(35 \mathrm{~A})$ & 6065 & 3875 & 3903 & 45 \\
$\mathrm{H}(36 \mathrm{~A})$ & 7773 & 4074 & 2764 & 55 \\
$\mathrm{H}(37 \mathrm{~A})$ & 9308 & 5048 & 3268 & 57 \\
$\mathrm{H}(38 \mathrm{~A})$ & 9053 & 5848 & 4855 & 42 \\
\hline
\end{tabular}




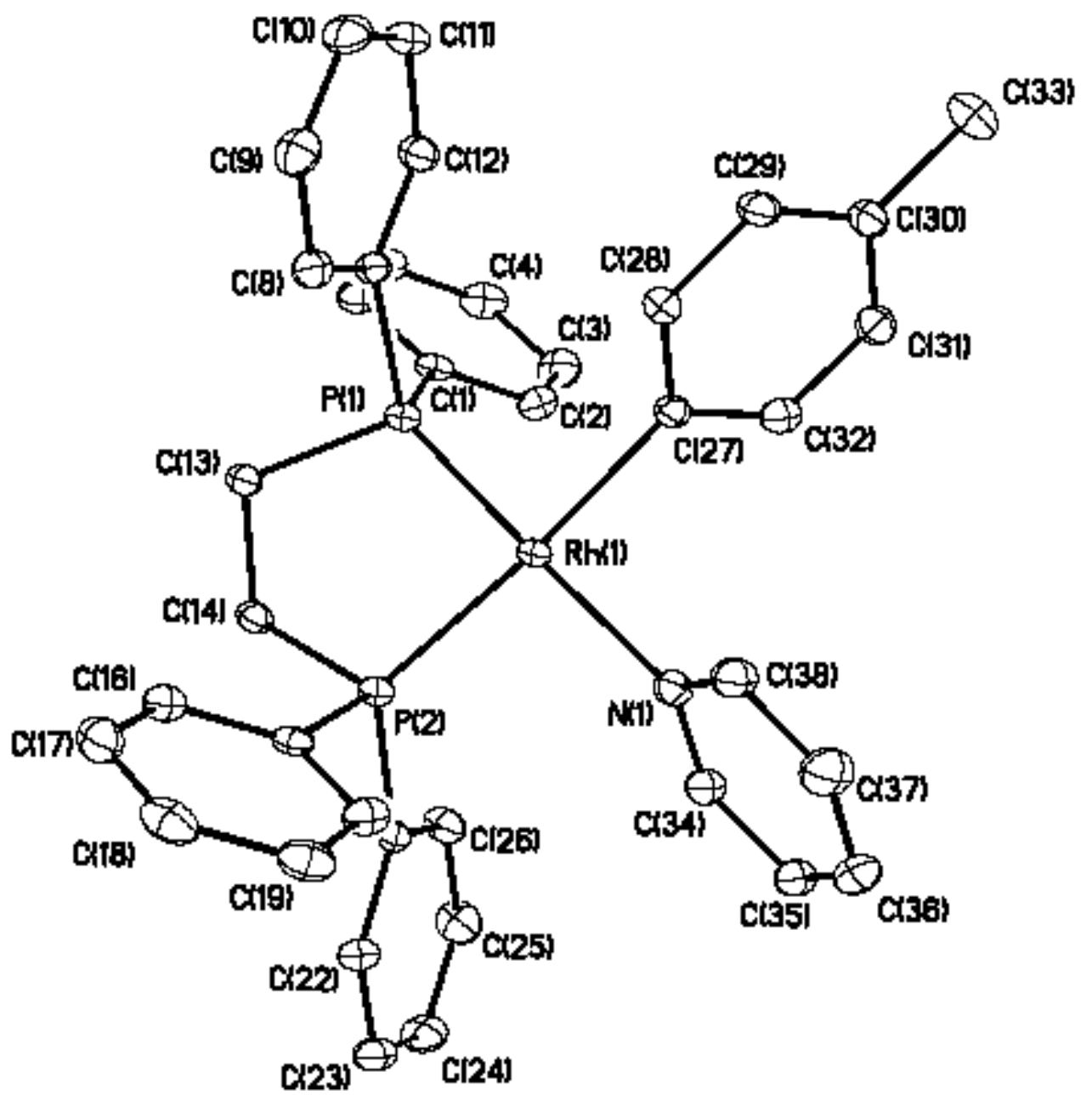

Figure S2. ORTEP diagram of (DPPE)Rh(pyr)(p-tol) (2).

Experimental Procedure for the X-ray Diffraction of $\mathbf{4}$ 
Data Collection

A red block crystal of C54H51N2O2P2Rh · C7H8, C61H59N2O2P2Rh, having approximate dimensions of $0.25 \times 0.25 \times 0.25 \mathrm{~mm}$ was mounted with epoxy cement on the tip of a fine glass fiber. All measurements were made on a Nonius KappaCCD diffractometer with graphite monochromated Mo-Ka radiation.

Cell constants and an orientation matrix for data collection corresponded to a primitive monoclinic cell with dimensions:

$$
\begin{array}{llll}
\mathrm{a}=20.443(4) \AA & \mathrm{a}=90 \mathrm{o} \\
\mathrm{b}=13.454(3) \AA & \mathrm{b}=110.31(3) \mathrm{o} \\
\mathrm{c}=20.069(4) \AA & \square=90 \mathrm{o} \\
\mathrm{V}=5176.4(18) \AA^{3} & &
\end{array}
$$

For $Z=4$ and F.W. $=1016.95$, the calculated density is $1.305 \mathrm{~g} / \mathrm{cm}^{3}$. Based on a statistical analysis of intensity distribution, and the successful solution and refinement of the structure, the space group was determined to be: $P 2_{1} / c$ (\#14)

The data were collected at a temperature of $173(2) \mathrm{K}$ to a maximum $2 \square$ value of $56.68^{\circ}$. Four omega scans consisting of 44, 44, 29, and 17 data frames, respectively, were collected with a frame width of $1.7^{\circ}$ and a detector-to-crystal distance, Dx, of $35.0 \mathrm{~mm}$. Each frame was exposed twice (for the purpose of de-zingering) for a total of 51 seconds. The data frames were processed and scaled using the DENZO software package. ${ }^{1}$

\section{$\underline{\text { Data Reduction }}$}

A total of 19766 reflections were collected of which 12809 were unique and observed $\left(\mathrm{R}_{\text {int }}=0.0471\right)$. The linear absorption coefficient, $\square$, for Mo-K $\square$ radiation is $4.37 \mathrm{~cm}^{-1}$ and no absorption correction was applied. The data were corrected for Lorentz and polarization effects.

$\underline{\text { Structure Solution and Refinement }}$

The structure was solved by direct methods and expanded using Fourier techniques ${ }^{2}$. The non-hydrogen atoms were refined anisotropically and hydrogen atoms were treated as idealized contributions. The final cycle of full-matrix least-squares refinement ${ }^{3}$ on $\mathrm{F}_{\text {was }}$ based on 12809 observed reflections (I > 2.00 $\square(\mathrm{I})$ ) and 641 variable parameters and converged with unweighted and weighted agreement factors of:

$$
\begin{gathered}
\mathrm{R}=\square\|\mathrm{Fo}|-| \mathrm{Fc}\| / \square|\mathrm{Fo}|=0.0505 \\
\mathrm{R}_{\mathrm{W}}=\left\{\square\left[\mathrm{w}\left(\mathrm{F}_{\mathrm{o}}{ }^{2}-\mathrm{F}_{\mathrm{c}}{ }^{2}\right)^{2}\right] / \square\left[\mathrm{w}\left(\mathrm{F}_{\mathrm{o}}{ }^{2}\right)^{2}\right]\right\}^{1 / 2}=0.1052
\end{gathered}
$$


The maximum and minimum peaks on the final difference Fourier map corresponded to 0.542 and $-0.567 \mathrm{e}^{-} / \AA^{3}$, respectively.

\section{REFERENCES}

(1) Z. Otwinowski and W. Minor, "Processing of X-Ray Diffraction Data Collected in Oscillation Mode," Methods in Enzymology, vol. 276: Macromolecular Crystallography, part A, 307-326, 1997, C.W. Carter, Jr. \& R.M. Sweet, Eds., Academic Press.

(2) Acta Cryst. A46 (1990) 467-473

(3) Least Squares function minimized:

$$
\square w\left(\mathrm{~F}_{\mathrm{o}}^{2}-\mathrm{F}_{\mathrm{c}}{ }^{2}\right)^{2}
$$

Table S6. Crystal data and structure refinement for 4. 
Empirical formula

Formula weight

Temperature

Wavelength

Crystal system

Space group

Unit cell dimensions

Volume

Z

Density (calculated)

Absorption coefficient

$\mathrm{F}(000)$

Crystal size

Theta range for data collection

Index ranges

Reflections collected

Independent reflections

Completeness to theta $=28.34^{\circ}$

Absorption correction

Max. and min. transmission

Refinement method

Data / restraints / parameters

Goodness-of-fit on $\mathrm{F}^{2}$

Final R indices [I $>2 \operatorname{sigma}(\mathrm{I})]$

$\mathrm{R}$ indices (all data)

Largest diff. peak and hole
$\mathrm{C}_{61} \mathrm{H}_{59} \mathrm{~N}_{2} \mathrm{O}_{2} \mathrm{P}_{2} \mathrm{Rh}$

1016.95

173(2) K

$0.71073 \AA$

Monoclinic

$\mathrm{P} 2(1) / \mathrm{c}$

$\mathrm{a}=20.443(4) \AA$

$\square=90^{\circ}$.

$\mathrm{b}=13.454(3) \AA$

$\square=110.31(3)^{\circ}$.

$\mathrm{c}=20.069(4) \AA$

$\square=90^{\circ}$.

5176.4(18) $\AA^{3}$

4

$1.305 \mathrm{~g} / \mathrm{cm}^{3}$

$4.37 \mathrm{~cm}^{-1}$

2120

$0.25 \times 0.25 \times 0.25 \mathrm{~mm}^{3}$

2.32 to $28.34^{\circ}$.

$-27<=\mathrm{h}<=27,-17<=\mathrm{k}<=17,-26<=\mathrm{l}<=26$

19766

$12809[\mathrm{R}(\mathrm{int})=0.0471]$

$99.1 \%$

None

0.8986 and 0.8986

Full-matrix least-squares on $\mathrm{F}^{2}$

12809 / 0 / 641

1.017

$\mathrm{R} 1=0.0505, \mathrm{wR} 2=0.1052$

$\mathrm{R} 1=0.1024, w R 2=0.1209$

0.542 and -0.567 e. $\AA^{-3}$ 
Table S7. Atomic coordinates ( $\left.\times 10^{4}\right)$ and equivalent isotropic displacement parameters $\left(\AA^{2} \times 10^{3}\right)$ for 4. $U(\mathrm{eq})$ is defined as one third of the trace of the orthogonalized $U^{\mathrm{ij}}$ tensor.

\begin{tabular}{|c|c|c|c|c|}
\hline & $\mathrm{x}$ & $\mathrm{y}$ & $\mathrm{z}$ & $\mathrm{U}(\mathrm{eq})$ \\
\hline $\mathrm{Rh}(1)$ & $7134(1)$ & $8529(1)$ & $3919(1)$ & $20(1)$ \\
\hline $\mathrm{P}(1)$ & $7520(1)$ & $8373(1)$ & $5087(1)$ & $22(1)$ \\
\hline $\mathrm{P}(2)$ & $6765(1)$ & 6990(1) & $3927(1)$ & $24(1)$ \\
\hline $\mathrm{O}(1)$ & $5584(1)$ & $12793(2)$ & $5104(1)$ & $46(1)$ \\
\hline $\mathrm{O}(2)$ & $6416(1)$ & $13866(2)$ & $5114(2)$ & $53(1)$ \\
\hline $\mathrm{N}(1)$ & $7477(1)$ & $10039(2)$ & $3905(1)$ & $22(1)$ \\
\hline $\mathrm{N}(2)$ & $6595(1)$ & $8638(2)$ & $2795(1)$ & $25(1)$ \\
\hline$C(1)$ & $8353(2)$ & $8893(2)$ & $5654(2)$ & $25(1)$ \\
\hline$C(2)$ & $8905(2)$ & $8297(3)$ & $6064(2)$ & $35(1)$ \\
\hline$C(3)$ & $9538(2)$ & $8737(3)$ & $6460(2)$ & $48(1)$ \\
\hline$C(4)$ & $9631(2)$ & $9747(3)$ & $6431(2)$ & $44(1)$ \\
\hline$C(5)$ & $9090(2)$ & $10330(3)$ & $6034(2)$ & $38(1)$ \\
\hline$C(6)$ & $8452(2)$ & $9916(2)$ & $5646(2)$ & $30(1)$ \\
\hline$C(7)$ & $6919(2)$ & $8842(2)$ & $5518(2)$ & $24(1)$ \\
\hline$C(8)$ & $7138(2)$ & $9227(2)$ & $6200(2)$ & $32(1)$ \\
\hline$C(9)$ & $6653(2)$ & $9565(3)$ & $6490(2)$ & $42(1)$ \\
\hline$C(10)$ & $5949(2)$ & $9515(3)$ & $6107(2)$ & $41(1)$ \\
\hline $\mathrm{C}(11)$ & $5724(2)$ & $9102(2)$ & $5436(2)$ & $34(1)$ \\
\hline$C(12)$ & $6206(2)$ & $8779(2)$ & $5142(2)$ & $26(1)$ \\
\hline$C(13)$ & $7566(2)$ & $7043(2)$ & $5329(2)$ & $29(1)$ \\
\hline$C(14)$ & $6901(2)$ & $6550(2)$ & $4842(2)$ & $29(1)$ \\
\hline$C(15)$ & $5842(2)$ & $6790(2)$ & $3414(2)$ & $28(1)$ \\
\hline$C(16)$ & $5315(2)$ & 6999(2) & $3690(2)$ & $36(1)$ \\
\hline $\mathrm{C}(17)$ & $4630(2)$ & 7049(3) & $3248(2)$ & $48(1)$ \\
\hline $\mathrm{C}(18)$ & $4444(2)$ & $6869(3)$ & $2529(2)$ & $50(1)$ \\
\hline$C(19)$ & 4952(2) & $6626(3)$ & $2256(2)$ & $42(1)$ \\
\hline $\mathrm{C}(20)$ & $5645(2)$ & $6587(2)$ & $2695(2)$ & $34(1)$ \\
\hline $\mathrm{C}(21)$ & $7205(2)$ & $6005(2)$ & $3612(2)$ & $26(1)$ \\
\hline $\mathrm{C}(22)$ & $7819(2)$ & $6219(2)$ & $3496(2)$ & $33(1)$ \\
\hline $\mathrm{C}(23)$ & $8180(2)$ & $5487(3)$ & $3286(2)$ & $45(1)$ \\
\hline$C(24)$ & $7925(2)$ & $4527(3)$ & $3185(2)$ & $47(1)$ \\
\hline
\end{tabular}




\begin{tabular}{|c|c|c|c|c|}
\hline$C(25)$ & $7319(2)$ & $4288(3)$ & $3299(2)$ & $45(1)$ \\
\hline$C(26)$ & $6955(2)$ & $5028(2)$ & $3516(2)$ & $37(1)$ \\
\hline$C(27)$ & $7164(2)$ & $10796(2)$ & $4122(2)$ & $23(1)$ \\
\hline $\mathrm{C}(28)$ & $6537(1)$ & $10598(2)$ & $4256(2)$ & $23(1)$ \\
\hline$C(29)$ & $6213(2)$ & $11298(2)$ & $4527(2)$ & $25(1)$ \\
\hline $\mathrm{C}(30)$ & $6465(2)$ & $12266(2)$ & $4667(2)$ & $27(1)$ \\
\hline $\mathrm{C}(31)$ & $7068(2)$ & $12490(2)$ & $4518(2)$ & $28(1)$ \\
\hline$C(32)$ & $7410(2)$ & $11795(2)$ & $4254(2)$ & $26(1)$ \\
\hline$C(33)$ & $6107(2)$ & $12972(2)$ & $4972(2)$ & $33(1)$ \\
\hline$C(34)$ & $6092(2)$ & $14580(3)$ & $5429(3)$ & $64(1)$ \\
\hline$C(35)$ & $8129(2)$ & $10288(2)$ & $3791(2)$ & $24(1)$ \\
\hline$C(36)$ & $8033(2)$ & $10990(2)$ & $3167(2)$ & $23(1)$ \\
\hline$C(37)$ & $7407(2)$ & $11095(2)$ & $2606(2)$ & $31(1)$ \\
\hline$C(38)$ & $7346(2)$ & $11744(2)$ & $2049(2)$ & $35(1)$ \\
\hline$C(39)$ & $7910(2)$ & $12312(2)$ & $2037(2)$ & $32(1)$ \\
\hline $\mathrm{C}(40)$ & $8534(2)$ & $12197(2)$ & $2593(2)$ & $33(1)$ \\
\hline $\mathrm{C}(41)$ & $8594(2)$ & $11553(2)$ & $3154(2)$ & $30(1)$ \\
\hline $\mathrm{C}(42)$ & $7839(2)$ & $13050(3)$ & $1445(2)$ & $47(1)$ \\
\hline $\mathrm{C}(43)$ & $8536(2)$ & $9363(2)$ & $3711(2)$ & $29(1)$ \\
\hline $\mathrm{C}(44)$ & $9071(2)$ & $9000(3)$ & 4291(2) & $40(1)$ \\
\hline $\mathrm{C}(45)$ & $9489(2)$ & $8224(3)$ & $4223(2)$ & $48(1)$ \\
\hline$C(46)$ & $9380(2)$ & $7786(3)$ & $3565(2)$ & $46(1)$ \\
\hline $\mathrm{C}(47)$ & $8843(2)$ & 8134(3) & 2992(2) & $41(1)$ \\
\hline $\mathrm{C}(48)$ & $8427(2)$ & $8923(2)$ & $3062(2)$ & $35(1)$ \\
\hline $\mathrm{C}(49)$ & $9864(2)$ & 6969(3) & $3489(3)$ & $67(1)$ \\
\hline $\mathrm{C}(50)$ & $6008(2)$ & $9188(2)$ & $2578(2)$ & $34(1)$ \\
\hline $\mathrm{C}(51)$ & $5553(2)$ & 9198(3) & 1883(2) & $44(1)$ \\
\hline $\mathrm{C}(52)$ & $5701(2)$ & $8633(3)$ & $1380(2)$ & $40(1)$ \\
\hline $\mathrm{C}(53)$ & 6311(2) & $8096(3)$ & 1592(2) & $36(1)$ \\
\hline $\mathrm{C}(54)$ & $6737(2)$ & $8113(2)$ & $2296(2)$ & $31(1)$ \\
\hline$C(55)$ & $9343(3)$ & $5446(4)$ & $6532(2)$ & $61(1)$ \\
\hline$C(57)$ & $8293(3)$ & $4534(3)$ & $5849(3)$ & $75(1)$ \\
\hline $\mathrm{C}(59)$ & $9051(2)$ & $5282(3)$ & $5279(2)$ & $52(1)$ \\
\hline $\mathrm{C}(61)$ & 10133(3) & $6235(5)$ & $5956(4)$ & $93(2)$ \\
\hline$C(56)$ & $8770(6)$ & 4949(6) & $6498(4)$ & $69(3)$ \\
\hline$C(58)$ & $8489(3)$ & 4721(4) & $5215(3)$ & $43(1)$ \\
\hline
\end{tabular}




\begin{tabular}{llllc}
$\mathrm{C}(60)$ & $9515(3)$ & $5686(4)$ & $5888(3)$ & $42(1)$ \\
$\mathrm{C}\left(56^{\prime}\right)$ & $9891(9)$ & $5803(9)$ & $6679(8)$ & $79(5)$ \\
$\mathrm{C}\left(58^{\prime}\right)$ & $9597(13)$ & $5807(13)$ & $5391(16)$ & $139(11)$ \\
$\mathrm{C}\left(60^{\prime}\right)$ & $8827(7)$ & $5044(10)$ & $6020(13)$ & $90(7)$ \\
\hline
\end{tabular}


Table S8. Bond lengths $[\AA]$ and angles $\left[{ }^{\circ}\right]$ for $\mathbf{4}$.

\begin{tabular}{|c|c|c|c|}
\hline $\mathrm{Rh}(1)-\mathrm{N}(2)$ & $2.145(3)$ & $\mathrm{C}(18)-\mathrm{C}(19)$ & $1.371(5)$ \\
\hline $\mathrm{Rh}(1)-\mathrm{N}(1)$ & $2.153(2)$ & $\mathrm{C}(19)-\mathrm{C}(20)$ & $1.385(5)$ \\
\hline $\mathrm{Rh}(1)-\mathrm{P}(2)$ & $2.2055(9)$ & $\mathrm{C}(21)-\mathrm{C}(22)$ & $1.386(4)$ \\
\hline $\mathrm{Rh}(1)-\mathrm{P}(1)$ & $2.2073(10)$ & $\mathrm{C}(21)-\mathrm{C}(26)$ & $1.399(4)$ \\
\hline $\mathrm{P}(1)-\mathrm{C}(1)$ & $1.828(3)$ & $\mathrm{C}(22)-\mathrm{C}(23)$ & $1.381(4)$ \\
\hline $\mathrm{P}(1)-\mathrm{C}(7)$ & $1.842(3)$ & $\mathrm{C}(23)-\mathrm{C}(24)$ & $1.382(5)$ \\
\hline $\mathrm{P}(1)-\mathrm{C}(13)$ & $1.848(3)$ & $\mathrm{C}(24)-\mathrm{C}(25)$ & $1.375(5)$ \\
\hline $\mathrm{P}(2)-\mathrm{C}(15)$ & $1.829(3)$ & $C(25)-C(26)$ & $1.399(5)$ \\
\hline $\mathrm{P}(2)-\mathrm{C}(21)$ & $1.832(3)$ & $\mathrm{C}(27)-\mathrm{C}(28)$ & $1.424(4)$ \\
\hline $\mathrm{P}(2)-\mathrm{C}(14)$ & $1.856(3)$ & $C(27)-C(32)$ & $1.427(4)$ \\
\hline $\mathrm{O}(1)-\mathrm{C}(33)$ & $1.210(4)$ & $\mathrm{C}(28)-\mathrm{C}(29)$ & $1.368(4)$ \\
\hline $\mathrm{O}(2)-\mathrm{C}(33)$ & $1.343(4)$ & C(29)-C(30) & $1.392(4)$ \\
\hline $\mathrm{O}(2)-\mathrm{C}(34)$ & $1.432(4)$ & $C(30)-C(31)$ & $1.399(4)$ \\
\hline $\mathrm{N}(1)-\mathrm{C}(27)$ & $1.353(4)$ & $\mathrm{C}(30)-\mathrm{C}(33)$ & $1.458(4)$ \\
\hline $\mathrm{N}(1)-\mathrm{C}(35)$ & $1.465(3)$ & $\mathrm{C}(31)-\mathrm{C}(32)$ & $1.379(4)$ \\
\hline $\mathrm{N}(2)-\mathrm{C}(54)$ & $1.337(4)$ & $\mathrm{C}(35)-\mathrm{C}(36)$ & $1.526(4)$ \\
\hline $\mathrm{N}(2)-\mathrm{C}(50)$ & $1.347(4)$ & $C(35)-C(43)$ & $1.536(4)$ \\
\hline$C(1)-C(6)$ & $1.392(4)$ & $\mathrm{C}(36)-\mathrm{C}(41)$ & $1.383(4)$ \\
\hline $\mathrm{C}(1)-\mathrm{C}(2)$ & $1.396(4)$ & $\mathrm{C}(36)-\mathrm{C}(37)$ & $1.389(4)$ \\
\hline $\mathrm{C}(2)-\mathrm{C}(3)$ & $1.394(5)$ & $\mathrm{C}(37)-\mathrm{C}(38)$ & $1.390(4)$ \\
\hline$C(3)-C(4)$ & $1.376(5)$ & $\mathrm{C}(38)-\mathrm{C}(39)$ & $1.390(4)$ \\
\hline$C(4)-C(5)$ & $1.363(5)$ & $C(39)-C(40)$ & $1.381(5)$ \\
\hline$C(5)-C(6)$ & $1.383(4)$ & $\mathrm{C}(39)-\mathrm{C}(42)$ & $1.515(4)$ \\
\hline$C(7)-C(8)$ & $1.384(4)$ & $\mathrm{C}(40)-\mathrm{C}(41)$ & $1.392(4)$ \\
\hline$C(7)-C(12)$ & $1.392(4)$ & $\mathrm{C}(43)-\mathrm{C}(48)$ & $1.376(4)$ \\
\hline $\mathrm{C}(8)-\mathrm{C}(9)$ & $1.388(4)$ & $\mathrm{C}(43)-\mathrm{C}(44)$ & $1.381(4)$ \\
\hline$C(9)-C(10)$ & $1.377(5)$ & $\mathrm{C}(44)-\mathrm{C}(45)$ & $1.386(5)$ \\
\hline$C(10)-C(11)$ & $1.380(5)$ & $\mathrm{C}(45)-\mathrm{C}(46)$ & $1.392(5)$ \\
\hline$C(11)-C(12)$ & $1.384(4)$ & $\mathrm{C}(46)-\mathrm{C}(47)$ & $1.366(5)$ \\
\hline$C(13)-C(14)$ & $1.523(4)$ & $\mathrm{C}(46)-\mathrm{C}(49)$ & $1.521(5)$ \\
\hline$C(15)-C(20)$ & $1.383(4)$ & $\mathrm{C}(47)-\mathrm{C}(48)$ & $1.398(5)$ \\
\hline$C(15)-C(16)$ & $1.399(4)$ & $\mathrm{C}(50)-\mathrm{C}(51)$ & $1.382(4)$ \\
\hline$C(16)-C(17)$ & $1.376(5)$ & $\mathrm{C}(51)-\mathrm{C}(52)$ & $1.378(5)$ \\
\hline$C(17)-C(18)$ & $1.380(5)$ & $C(52)-C(53)$ & $1.376(5)$ \\
\hline
\end{tabular}




\begin{tabular}{|c|c|c|c|}
\hline $\mathrm{C}(53)-\mathrm{C}(54)$ & $1.378(4)$ & $\mathrm{C}(27)-\mathrm{N}(1)-\mathrm{Rh}(1)$ & $120.90(18)$ \\
\hline$C(55)-C\left(56^{\prime}\right)$ & $1.158(17)$ & $\mathrm{C}(35)-\mathrm{N}(1)-\mathrm{Rh}(1)$ & $122.43(17)$ \\
\hline$C(55)-C(56)$ & $1.330(11)$ & $\mathrm{C}(54)-\mathrm{N}(2)-\mathrm{C}(50)$ & $116.8(3)$ \\
\hline $\mathrm{C}(55)-\mathrm{C}\left(60^{\prime}\right)$ & $1.308(18)$ & $\mathrm{C}(54)-\mathrm{N}(2)-\mathrm{Rh}(1)$ & $125.9(2)$ \\
\hline $\mathrm{C}(55)-\mathrm{C}(60)$ & $1.488(9)$ & $\mathrm{C}(50)-\mathrm{N}(2)-\mathrm{Rh}(1)$ & $116.8(2)$ \\
\hline $\mathrm{C}(57)-\mathrm{C}\left(60^{\prime}\right)$ & $1.232(15)$ & $\mathrm{C}(6)-\mathrm{C}(1)-\mathrm{C}(2)$ & $119.0(3)$ \\
\hline$C(57)-C(56)$ & $1.441(11)$ & $\mathrm{C}(6)-\mathrm{C}(1)-\mathrm{P}(1)$ & $118.6(2)$ \\
\hline $\mathrm{C}(57)-\mathrm{C}(58)$ & $1.482(8)$ & $\mathrm{C}(2)-\mathrm{C}(1)-\mathrm{P}(1)$ & $122.3(2)$ \\
\hline $\mathrm{C}(59)-\mathrm{C}\left(58^{\prime}\right)$ & $1.27(2)$ & $C(3)-C(2)-C(1)$ & $119.5(3)$ \\
\hline$C(59)-C(58)$ & $1.343(7)$ & $C(4)-C(3)-C(2)$ & $120.7(3)$ \\
\hline $\mathrm{C}(59)-\mathrm{C}(60)$ & $1.372(7)$ & $C(5)-C(4)-C(3)$ & $119.8(3)$ \\
\hline $\mathrm{C}(59)-\mathrm{C}\left(60^{\prime}\right)$ & $1.73(3)$ & $C(4)-C(5)-C(6)$ & $120.8(3)$ \\
\hline $\mathrm{C}(61)-\mathrm{C}\left(58^{\prime}\right)$ & $1.40(3)$ & $C(5)-C(6)-C(1)$ & $120.3(3)$ \\
\hline$C(61)-C(60)$ & $1.429(9)$ & $\mathrm{C}(8)-\mathrm{C}(7)-\mathrm{C}(12)$ & $118.5(3)$ \\
\hline \multirow[t]{2}{*}{$C(61)-C\left(56^{\prime}\right)$} & $1.785(19)$ & $\mathrm{C}(8)-\mathrm{C}(7)-\mathrm{P}(1)$ & $123.6(2)$ \\
\hline & & $\mathrm{C}(12)-\mathrm{C}(7)-\mathrm{P}(1)$ & $117.9(2)$ \\
\hline $\mathrm{N}(2)-\mathrm{Rh}(1)-\mathrm{N}(1)$ & $88.44(9)$ & $\mathrm{C}(7)-\mathrm{C}(8)-\mathrm{C}(9)$ & $120.3(3)$ \\
\hline $\mathrm{N}(2)-\mathrm{Rh}(1)-\mathrm{P}(2)$ & $91.09(7)$ & $\mathrm{C}(10)-\mathrm{C}(9)-\mathrm{C}(8)$ & $120.7(3)$ \\
\hline $\mathrm{N}(1)-\mathrm{Rh}(1)-\mathrm{P}(2)$ & $179.14(7)$ & $\mathrm{C}(9)-\mathrm{C}(10)-\mathrm{C}(11)$ & 119.6(3) \\
\hline $\mathrm{N}(2)-\mathrm{Rh}(1)-\mathrm{P}(1)$ & $170.71(7)$ & $C(12)-C(11)-C(10)$ & $119.8(3)$ \\
\hline $\mathrm{N}(1)-\mathrm{Rh}(1)-\mathrm{P}(1)$ & $96.09(7)$ & $C(11)-C(12)-C(7)$ & $121.1(3)$ \\
\hline $\mathrm{P}(2)-\mathrm{Rh}(1)-\mathrm{P}(1)$ & $84.27(3)$ & $\mathrm{C}(14)-\mathrm{C}(13)-\mathrm{P}(1)$ & $107.5(2)$ \\
\hline $\mathrm{C}(1)-\mathrm{P}(1)-\mathrm{C}(7)$ & $101.84(14)$ & $\mathrm{C}(13)-\mathrm{C}(14)-\mathrm{P}(2)$ & $106.8(2)$ \\
\hline $\mathrm{C}(1)-\mathrm{P}(1)-\mathrm{C}(13)$ & $104.80(14)$ & $C(20)-C(15)-C(16)$ & $117.9(3)$ \\
\hline $\mathrm{C}(7)-\mathrm{P}(1)-\mathrm{C}(13)$ & $100.93(14)$ & $\mathrm{C}(20)-\mathrm{C}(15)-\mathrm{P}(2)$ & $119.1(2)$ \\
\hline $\mathrm{C}(1)-\mathrm{P}(1)-\mathrm{Rh}(1)$ & $122.49(10)$ & $\mathrm{C}(16)-\mathrm{C}(15)-\mathrm{P}(2)$ & $122.2(2)$ \\
\hline $\mathrm{C}(7)-\mathrm{P}(1)-\mathrm{Rh}(1)$ & $114.53(10)$ & $C(17)-C(16)-C(15)$ & $120.4(3)$ \\
\hline $\mathrm{C}(13)-\mathrm{P}(1)-\mathrm{Rh}(1)$ & $109.77(10)$ & $C(16)-C(17)-C(18)$ & $121.0(4)$ \\
\hline $\mathrm{C}(15)-\mathrm{P}(2)-\mathrm{C}(21)$ & $103.51(14)$ & $C(19)-C(18)-C(17)$ & 119.1(3) \\
\hline $\mathrm{C}(15)-\mathrm{P}(2)-\mathrm{C}(14)$ & $106.33(15)$ & $C(18)-C(19)-C(20)$ & $120.3(3)$ \\
\hline $\mathrm{C}(21)-\mathrm{P}(2)-\mathrm{C}(14)$ & $100.78(14)$ & $C(15)-C(20)-C(19)$ & $121.2(3)$ \\
\hline $\mathrm{C}(15)-\mathrm{P}(2)-\mathrm{Rh}(1)$ & $114.99(10)$ & $C(22)-C(21)-C(26)$ & $118.7(3)$ \\
\hline $\mathrm{C}(21)-\mathrm{P}(2)-\mathrm{Rh}(1)$ & $117.83(10)$ & $\mathrm{C}(22)-\mathrm{C}(21)-\mathrm{P}(2)$ & $119.4(2)$ \\
\hline $\mathrm{C}(14)-\mathrm{P}(2)-\mathrm{Rh}(1)$ & $111.87(10)$ & $\mathrm{C}(26)-\mathrm{C}(21)-\mathrm{P}(2)$ & $121.9(2)$ \\
\hline $\mathrm{C}(33)-\mathrm{O}(2)-\mathrm{C}(34)$ & $116.0(3)$ & $\mathrm{C}(23)-\mathrm{C}(22)-\mathrm{C}(21)$ & $121.0(3)$ \\
\hline $\mathrm{C}(27)-\mathrm{N}(1)-\mathrm{C}(35)$ & $115.7(2)$ & $\mathrm{C}(22)-\mathrm{C}(23)-\mathrm{C}(24)$ & $119.8(3)$ \\
\hline
\end{tabular}




\begin{tabular}{|c|c|c|c|}
\hline$C(25)-C(24)-C(23)$ & $120.6(3)$ & $\mathrm{C}(45)-\mathrm{C}(46)-\mathrm{C}(49)$ & $120.3(4)$ \\
\hline$C(24)-C(25)-C(26)$ & $119.5(3)$ & $\mathrm{C}(46)-\mathrm{C}(47)-\mathrm{C}(48)$ & $121.0(4)$ \\
\hline$C(25)-C(26)-C(21)$ & $120.3(3)$ & $\mathrm{C}(43)-\mathrm{C}(48)-\mathrm{C}(47)$ & 121.2(3) \\
\hline $\mathrm{N}(1)-\mathrm{C}(27)-\mathrm{C}(28)$ & $118.5(3)$ & $\mathrm{N}(2)-\mathrm{C}(50)-\mathrm{C}(51)$ & $122.8(3)$ \\
\hline $\mathrm{N}(1)-\mathrm{C}(27)-\mathrm{C}(32)$ & $126.5(3)$ & $C(52)-C(51)-C(50)$ & $119.5(3)$ \\
\hline $\mathrm{C}(28)-\mathrm{C}(27)-\mathrm{C}(32)$ & 115.1(3) & $\mathrm{C}(53)-\mathrm{C}(52)-\mathrm{C}(51)$ & $117.9(3)$ \\
\hline $\mathrm{C}(29)-\mathrm{C}(28)-\mathrm{C}(27)$ & $122.5(3)$ & $\mathrm{C}(52)-\mathrm{C}(53)-\mathrm{C}(54)$ & $119.4(3)$ \\
\hline$C(28)-C(29)-C(30)$ & $121.9(3)$ & $\mathrm{N}(2)-\mathrm{C}(54)-\mathrm{C}(53)$ & $123.5(3)$ \\
\hline$C(29)-C(30)-C(31)$ & $116.7(3)$ & $C\left(56^{\prime}\right)-C(55)-C(56)$ & $167.9(10)$ \\
\hline$C(29)-C(30)-C(33)$ & 119.4(3) & $\mathrm{C}\left(56^{\prime}\right)-\mathrm{C}(55)-\mathrm{C}\left(60^{\prime}\right)$ & $144.8(14)$ \\
\hline$C(31)-C(30)-C(33)$ & $123.9(3)$ & $\mathrm{C}(56)-\mathrm{C}(55)-\mathrm{C}\left(60^{\prime}\right)$ & $45.2(10)$ \\
\hline $\mathrm{C}(32)-\mathrm{C}(31)-\mathrm{C}(30)$ & $122.6(3)$ & $\mathrm{C}\left(56^{\prime}\right)-\mathrm{C}(55)-\mathrm{C}(60)$ & 68.4(9) \\
\hline $\mathrm{C}(31)-\mathrm{C}(32)-\mathrm{C}(27)$ & $121.1(3)$ & $\mathrm{C}(56)-\mathrm{C}(55)-\mathrm{C}(60)$ & $122.3(6)$ \\
\hline $\mathrm{O}(1)-\mathrm{C}(33)-\mathrm{O}(2)$ & $121.2(3)$ & $\mathrm{C}\left(60^{\prime}\right)-\mathrm{C}(55)-\mathrm{C}(60)$ & 77.1(11) \\
\hline $\mathrm{O}(1)-\mathrm{C}(33)-\mathrm{C}(30)$ & $124.9(3)$ & $\mathrm{C}\left(60^{\prime}\right)-\mathrm{C}(57)-\mathrm{C}(56)$ & $43.7(12)$ \\
\hline $\mathrm{O}(2)-\mathrm{C}(33)-\mathrm{C}(30)$ & $113.9(3)$ & $\mathrm{C}\left(60^{\prime}\right)-\mathrm{C}(57)-\mathrm{C}(58)$ & $70.5(13)$ \\
\hline $\mathrm{N}(1)-\mathrm{C}(35)-\mathrm{C}(36)$ & $113.9(2)$ & $C(56)-C(57)-C(58)$ & $114.2(6)$ \\
\hline $\mathrm{N}(1)-\mathrm{C}(35)-\mathrm{C}(43)$ & $112.7(2)$ & $\mathrm{C}\left(58^{\prime}\right)-\mathrm{C}(59)-\mathrm{C}(58)$ & $175.6(14)$ \\
\hline$C(36)-C(35)-C(43)$ & 109.6(2) & $\mathrm{C}\left(58^{\prime}\right)-\mathrm{C}(59)-\mathrm{C}(60)$ & $48.0(12)$ \\
\hline $\mathrm{C}(41)-\mathrm{C}(36)-\mathrm{C}(37)$ & $117.7(3)$ & $\mathrm{C}(58)-\mathrm{C}(59)-\mathrm{C}(60)$ & $127.7(6)$ \\
\hline $\mathrm{C}(41)-\mathrm{C}(36)-\mathrm{C}(35)$ & 119.0(3) & $\mathrm{C}\left(58^{\prime}\right)-\mathrm{C}(59)-\mathrm{C}\left(60^{\prime}\right)$ & $115.4(14)$ \\
\hline $\mathrm{C}(37)-\mathrm{C}(36)-\mathrm{C}(35)$ & $123.2(3)$ & $\mathrm{C}(58)-\mathrm{C}(59)-\mathrm{C}\left(60^{\prime}\right)$ & $60.3(6)$ \\
\hline $\mathrm{C}(36)-\mathrm{C}(37)-\mathrm{C}(38)$ & 121.1(3) & $\mathrm{C}(60)-\mathrm{C}(59)-\mathrm{C}\left(60^{\prime}\right)$ & $67.5(6)$ \\
\hline $\mathrm{C}(39)-\mathrm{C}(38)-\mathrm{C}(37)$ & $121.0(3)$ & $\mathrm{C}\left(58^{\prime}\right)-\mathrm{C}(61)-\mathrm{C}(60)$ & $44.9(7)$ \\
\hline $\mathrm{C}(40)-\mathrm{C}(39)-\mathrm{C}(38)$ & $117.7(3)$ & $\mathrm{C}\left(58^{\prime}\right)-\mathrm{C}(61)-\mathrm{C}\left(56^{\prime}\right)$ & $99.5(10)$ \\
\hline$C(40)-C(39)-C(42)$ & $121.1(3)$ & $\mathrm{C}(60)-\mathrm{C}(61)-\mathrm{C}\left(56^{\prime}\right)$ & $54.8(6)$ \\
\hline $\mathrm{C}(38)-\mathrm{C}(39)-\mathrm{C}(42)$ & $121.2(3)$ & $\mathrm{C}(55)-\mathrm{C}(56)-\mathrm{C}(57)$ & $123.3(6)$ \\
\hline $\mathrm{C}(39)-\mathrm{C}(40)-\mathrm{C}(41)$ & 121.3(3) & $\mathrm{C}(59)-\mathrm{C}(58)-\mathrm{C}(57)$ & $119.4(5)$ \\
\hline $\mathrm{C}(36)-\mathrm{C}(41)-\mathrm{C}(40)$ & 121.1(3) & $\mathrm{C}(59)-\mathrm{C}(60)-\mathrm{C}(61)$ & 127.3(7) \\
\hline $\mathrm{C}(48)-\mathrm{C}(43)-\mathrm{C}(44)$ & $117.8(3)$ & $C(59)-C(60)-C(55)$ & $112.9(6)$ \\
\hline $\mathrm{C}(48)-\mathrm{C}(43)-\mathrm{C}(35)$ & $122.1(3)$ & $\mathrm{C}(61)-\mathrm{C}(60)-\mathrm{C}(55)$ & $119.8(6)$ \\
\hline $\mathrm{C}(44)-\mathrm{C}(43)-\mathrm{C}(35)$ & $119.9(3)$ & $\mathrm{C}(55)-\mathrm{C}\left(56^{\prime}\right)-\mathrm{C}(61)$ & $116.4(11)$ \\
\hline$C(43)-C(44)-C(45)$ & $121.3(4)$ & $\mathrm{C}(59)-\mathrm{C}\left(58^{\prime}\right)-\mathrm{C}(61)$ & $139.8(19)$ \\
\hline$C(44)-C(45)-C(46)$ & $120.7(4)$ & $\mathrm{C}(57)-\mathrm{C}\left(60^{\prime}\right)-\mathrm{C}(55)$ & $148(2)$ \\
\hline$C(47)-C(46)-C(45)$ & $118.1(3)$ & $\mathrm{C}(57)-\mathrm{C}\left(60^{\prime}\right)-\mathrm{C}(59)$ & $109.7(16)$ \\
\hline $\mathrm{C}(47)-\mathrm{C}(46)-\mathrm{C}(49)$ & $121.6(4)$ & $\mathrm{C}(55)-\mathrm{C}\left(60^{\prime}\right)-\mathrm{C}(59)$ & $102.4(12)$ \\
\hline
\end{tabular}


Table S9. Anisotropic displacement parameters $\left(\AA^{2} \times 10^{3}\right)$ for 4 . The anisotropic displacement factor exponent takes the form: $-2 \square^{2}\left[h^{2} a^{* 2} U^{11}+\ldots+2 h k a^{*} b^{*} U^{12}\right]$

\begin{tabular}{|c|c|c|c|c|c|c|}
\hline & $\mathrm{U}^{11}$ & $\mathrm{U}^{22}$ & $\mathrm{U}^{33}$ & $\mathrm{U}^{23}$ & $\mathrm{U}^{13}$ & $\mathrm{U}^{12}$ \\
\hline $\mathrm{Rh}(1)$ & $23(1)$ & $19(1)$ & $19(1)$ & $0(1)$ & $8(1)$ & $-1(1)$ \\
\hline $\mathrm{P}(1)$ & $22(1)$ & $22(1)$ & $21(1)$ & $0(1)$ & $8(1)$ & $1(1)$ \\
\hline $\mathrm{P}(2)$ & $29(1)$ & $20(1)$ & $23(1)$ & $-1(1)$ & $10(1)$ & $-3(1)$ \\
\hline $\mathrm{O}(1)$ & $44(2)$ & $45(2)$ & $60(2)$ & $-12(1)$ & $31(1)$ & $2(1)$ \\
\hline $\mathrm{O}(2)$ & $76(2)$ & $25(1)$ & $82(2)$ & $-11(1)$ & $60(2)$ & $-4(1)$ \\
\hline $\mathrm{N}(1)$ & $24(1)$ & $16(1)$ & $28(1)$ & $1(1)$ & $12(1)$ & $-1(1)$ \\
\hline $\mathrm{N}(2)$ & $29(1)$ & $23(1)$ & $21(1)$ & $2(1)$ & $7(1)$ & $-4(1)$ \\
\hline $\mathrm{C}(1)$ & $23(2)$ & $33(2)$ & $22(2)$ & $-3(1)$ & $10(1)$ & $1(1)$ \\
\hline $\mathrm{C}(2)$ & $28(2)$ & $37(2)$ & $38(2)$ & $7(2)$ & $10(2)$ & $1(2)$ \\
\hline$C(3)$ & $21(2)$ & $66(3)$ & $49(2)$ & $12(2)$ & $4(2)$ & $8(2)$ \\
\hline$C(4)$ & $25(2)$ & $63(3)$ & $40(2)$ & $-14(2)$ & $8(2)$ & $-13(2)$ \\
\hline$C(5)$ & $33(2)$ & $38(2)$ & $44(2)$ & $-11(2)$ & $16(2)$ & $-6(2)$ \\
\hline$C(6)$ & $28(2)$ & $35(2)$ & $29(2)$ & $-6(2)$ & $11(1)$ & $-2(1)$ \\
\hline$C(7)$ & $29(2)$ & $23(2)$ & $23(2)$ & $1(1)$ & $13(1)$ & $-1(1)$ \\
\hline $\mathrm{C}(8)$ & $33(2)$ & $37(2)$ & $26(2)$ & $-5(2)$ & $12(2)$ & $-2(2)$ \\
\hline$C(9)$ & $47(2)$ & $49(2)$ & $36(2)$ & $-14(2)$ & $23(2)$ & $-3(2)$ \\
\hline $\mathrm{C}(10)$ & $41(2)$ & $44(2)$ & $48(2)$ & $3(2)$ & $30(2)$ & $8(2)$ \\
\hline $\mathrm{C}(11)$ & $24(2)$ & $40(2)$ & $41(2)$ & $11(2)$ & $16(2)$ & $1(2)$ \\
\hline $\mathrm{C}(12)$ & $28(2)$ & $29(2)$ & $23(2)$ & $3(1)$ & $10(1)$ & $-3(1)$ \\
\hline $\mathrm{C}(13)$ & $36(2)$ & $25(2)$ & $26(2)$ & $3(1)$ & $12(2)$ & $3(1)$ \\
\hline$C(14)$ & $41(2)$ & $23(2)$ & $28(2)$ & $1(1)$ & $17(2)$ & $-3(1)$ \\
\hline$C(15)$ & $29(2)$ & $23(2)$ & $31(2)$ & $-2(1)$ & $10(2)$ & $-4(1)$ \\
\hline$C(16)$ & $33(2)$ & $39(2)$ & $37(2)$ & $-8(2)$ & $15(2)$ & $-10(2)$ \\
\hline $\mathrm{C}(17)$ & $29(2)$ & $51(2)$ & $68(3)$ & $-10(2)$ & $20(2)$ & $-7(2)$ \\
\hline$C(18)$ & $33(2)$ & $55(2)$ & $54(3)$ & $-4(2)$ & $5(2)$ & $-11(2)$ \\
\hline$C(19)$ & $36(2)$ & $53(2)$ & $31(2)$ & $-4(2)$ & $5(2)$ & $-16(2)$ \\
\hline$C(20)$ & $35(2)$ & $37(2)$ & $31(2)$ & $-1(2)$ & $11(2)$ & $-10(2)$ \\
\hline$C(21)$ & $35(2)$ & $24(2)$ & $20(2)$ & $-3(1)$ & $8(1)$ & $-1(1)$ \\
\hline $\mathrm{C}(22)$ & $37(2)$ & $27(2)$ & $32(2)$ & $0(2)$ & $8(2)$ & $1(2)$ \\
\hline$C(23)$ & $42(2)$ & $53(2)$ & $42(2)$ & $-2(2)$ & $17(2)$ & $15(2)$ \\
\hline$C(24)$ & $62(3)$ & $38(2)$ & $40(2)$ & $-5(2)$ & $14(2)$ & $22(2)$ \\
\hline
\end{tabular}




\begin{tabular}{|c|c|c|c|c|c|c|}
\hline$C(25)$ & $72(3)$ & $22(2)$ & $35(2)$ & $0(2)$ & $11(2)$ & $4(2)$ \\
\hline$C(26)$ & $53(2)$ & $27(2)$ & $31(2)$ & $-2(2)$ & $15(2)$ & $-2(2)$ \\
\hline$C(27)$ & $24(2)$ & $23(2)$ & $21(2)$ & 2(1) & $7(1)$ & $1(1)$ \\
\hline$C(28)$ & $23(2)$ & $22(2)$ & $23(2)$ & 2(1) & $8(1)$ & $1(1)$ \\
\hline$C(29)$ & $23(2)$ & $28(2)$ & $26(2)$ & 2(1) & $10(1)$ & 1(1) \\
\hline$C(30)$ & $27(2)$ & $29(2)$ & $27(2)$ & $2(1)$ & 11(1) & $5(1)$ \\
\hline $\mathrm{C}(31)$ & $33(2)$ & $22(2)$ & $31(2)$ & $0(1)$ & $15(2)$ & $-1(1)$ \\
\hline$C(32)$ & $28(2)$ & $25(2)$ & $29(2)$ & $0(1)$ & $14(1)$ & $-3(1)$ \\
\hline$C(33)$ & $40(2)$ & $33(2)$ & $29(2)$ & $0(2)$ & $17(2)$ & $6(2)$ \\
\hline$C(34)$ & $93(3)$ & $32(2)$ & $99(4)$ & $-10(2)$ & 74(3) & $5(2)$ \\
\hline$C(35)$ & $24(2)$ & $24(2)$ & $26(2)$ & $1(1)$ & $13(1)$ & $-2(1)$ \\
\hline$C(36)$ & $28(2)$ & $21(2)$ & $25(2)$ & $1(1)$ & $15(1)$ & $4(1)$ \\
\hline$C(37)$ & $28(2)$ & $30(2)$ & $39(2)$ & $3(2)$ & $16(2)$ & $-6(1)$ \\
\hline$C(38)$ & $32(2)$ & $41(2)$ & $31(2)$ & $10(2)$ & $9(2)$ & $5(2)$ \\
\hline$C(39)$ & $42(2)$ & $28(2)$ & $31(2)$ & $1(2)$ & $18(2)$ & $-2(2)$ \\
\hline $\mathrm{C}(40)$ & $35(2)$ & $27(2)$ & $42(2)$ & $2(2)$ & $20(2)$ & $-6(2)$ \\
\hline $\mathrm{C}(41)$ & $24(2)$ & $32(2)$ & $34(2)$ & $2(2)$ & 11(1) & $0(1)$ \\
\hline$C(42)$ & $65(3)$ & $42(2)$ & $37(2)$ & $9(2)$ & $21(2)$ & $-6(2)$ \\
\hline$C(43)$ & $32(2)$ & $25(2)$ & $37(2)$ & $6(2)$ & $20(2)$ & $1(1)$ \\
\hline$C(44)$ & $39(2)$ & $44(2)$ & $43(2)$ & $13(2)$ & $23(2)$ & $16(2)$ \\
\hline$C(45)$ & $46(2)$ & $50(2)$ & $55(3)$ & $20(2)$ & $27(2)$ & $17(2)$ \\
\hline$C(46)$ & $47(2)$ & $29(2)$ & $79(3)$ & $7(2)$ & $43(2)$ & $6(2)$ \\
\hline $\mathrm{C}(47)$ & $42(2)$ & $34(2)$ & $54(2)$ & $-2(2)$ & $27(2)$ & $-1(2)$ \\
\hline$C(48)$ & $35(2)$ & $29(2)$ & $45(2)$ & $-1(2)$ & $19(2)$ & $-1(2)$ \\
\hline$C(49)$ & $64(3)$ & $42(2)$ & $115(4)$ & $0(3)$ & $56(3)$ & $16(2)$ \\
\hline$C(50)$ & $39(2)$ & $30(2)$ & $32(2)$ & $-2(2)$ & $9(2)$ & $-1(2)$ \\
\hline$C(51)$ & $39(2)$ & $47(2)$ & $37(2)$ & $11(2)$ & $2(2)$ & $3(2)$ \\
\hline$C(52)$ & $49(2)$ & $41(2)$ & $22(2)$ & $3(2)$ & $3(2)$ & $-14(2)$ \\
\hline$C(53)$ & $54(2)$ & $34(2)$ & $23(2)$ & $-4(2)$ & $15(2)$ & $-10(2)$ \\
\hline$C(54)$ & $39(2)$ & $29(2)$ & $28(2)$ & $2(2)$ & $14(2)$ & $-1(2)$ \\
\hline$C(55)$ & $70(3)$ & $49(3)$ & $49(3)$ & $-7(2)$ & $2(3)$ & $15(3)$ \\
\hline$C(57)$ & $93(4)$ & $48(3)$ & $76(4)$ & $3(3)$ & $17(3)$ & $10(3)$ \\
\hline$C(59)$ & $70(3)$ & $41(2)$ & $39(2)$ & $3(2)$ & $10(2)$ & $16(2)$ \\
\hline$C(61)$ & $59(3)$ & $79(4)$ & $124(5)$ & $-38(4)$ & $8(3)$ & $7(3)$ \\
\hline$C(56)$ & $130(9)$ & $40(4)$ & $53(5)$ & $12(4)$ & $51(6)$ & $22(5)$ \\
\hline $\mathrm{C}(58)$ & $40(3)$ & $36(3)$ & $45(4)$ & $9(3)$ & $6(3)$ & $7(3)$ \\
\hline
\end{tabular}




\begin{tabular}{lcccccc}
$\mathrm{C}(60)$ & $43(4)$ & $27(3)$ & $52(4)$ & $-6(3)$ & $12(3)$ & $13(3)$ \\
$\mathrm{C}\left(56^{\prime}\right)$ & $104(12)$ & $33(7)$ & $74(11)$ & $-12(7)$ & $0(10)$ & $12(8)$ \\
$\mathrm{C}\left(58^{\prime}\right)$ & $180(20)$ & $56(11)$ & $280(30)$ & $83(15)$ & $200(20)$ & $76(13)$ \\
$\mathrm{C}\left(60^{\prime}\right)$ & $27(8)$ & $27(7)$ & $190(20)$ & $-9(12)$ & $4(11)$ & $15(6)$ \\
\hline
\end{tabular}


Table S10. Hydrogen coordinates ( $\left.\times 10^{4}\right)$ and isotropic displacement parameters $\left(\AA^{2} \times 10^{3}\right)$ for 4 .

\begin{tabular}{|c|c|c|c|c|}
\hline & $\mathrm{x}$ & $\mathrm{y}$ & z & $\mathrm{U}(\mathrm{eq})$ \\
\hline $\mathrm{H}(2 \mathrm{~A})$ & 8849 & 7597 & 6074 & 42 \\
\hline $\mathrm{H}(3 \mathrm{~A})$ & 9909 & 8336 & 6752 & 57 \\
\hline $\mathrm{H}(4 \mathrm{~A})$ & 10070 & 10038 & 6687 & 53 \\
\hline $\mathrm{H}(5 \mathrm{~A})$ & 9153 & 11029 & 6023 & 45 \\
\hline $\mathrm{H}(6 \mathrm{~A})$ & 8080 & 10331 & 5372 & 36 \\
\hline $\mathrm{H}(8 \mathrm{~A})$ & 7621 & 9259 & 6471 & 38 \\
\hline $\mathrm{H}(9 \mathrm{~A})$ & 6809 & 9834 & 6956 & 50 \\
\hline $\mathrm{H}(10 \mathrm{~A})$ & 5621 & 9763 & 6305 & 49 \\
\hline $\mathrm{H}(11 \mathrm{~A})$ & 5238 & 9040 & 5177 & 41 \\
\hline $\mathrm{H}(12 \mathrm{~A})$ & 6048 & 8510 & 4675 & 32 \\
\hline $\mathrm{H}(13 \mathrm{~A})$ & 7979 & 6730 & 5267 & 34 \\
\hline $\mathrm{H}(13 \mathrm{~B})$ & 7603 & 6968 & 5831 & 34 \\
\hline $\mathrm{H}(14 \mathrm{~A})$ & 6500 & 6738 & 4983 & 35 \\
\hline $\mathrm{H}(14 \mathrm{~B})$ & 6950 & 5817 & 4868 & 35 \\
\hline $\mathrm{H}(16 \mathrm{~A})$ & 5432 & 7108 & 4185 & 43 \\
\hline $\mathrm{H}(17 \mathrm{~A})$ & 4279 & 7210 & 3440 & 58 \\
\hline $\mathrm{H}(18 \mathrm{~A})$ & 3969 & 6913 & 2226 & 60 \\
\hline $\mathrm{H}(19 \mathrm{~A})$ & 4829 & 6484 & 1764 & 50 \\
\hline $\mathrm{H}(20 \mathrm{~A})$ & 5992 & 6418 & 2499 & 41 \\
\hline $\mathrm{H}(22 \mathrm{~A})$ & 7995 & 6879 & 3561 & 39 \\
\hline $\mathrm{H}(23 \mathrm{~A})$ & 8602 & 5644 & 3211 & 54 \\
\hline $\mathrm{H}(24 \mathrm{~A})$ & 8171 & 4026 & 3035 & 57 \\
\hline $\mathrm{H}(25 \mathrm{~A})$ & 7148 & 3626 & 3232 & 54 \\
\hline $\mathrm{H}(26 \mathrm{~A})$ & 6537 & 4866 & 3598 & 45 \\
\hline $\mathrm{H}(28 \mathrm{~A})$ & 6336 & 9954 & 4153 & 27 \\
\hline $\mathrm{H}(29 \mathrm{~A})$ & 5805 & 11119 & 4622 & 30 \\
\hline $\mathrm{H}(31 \mathrm{~A})$ & 7249 & 13147 & 4602 & 34 \\
\hline $\mathrm{H}(32 \mathrm{~A})$ & 7817 & 11985 & 4159 & 31 \\
\hline $\mathrm{H}(34 \mathrm{~A})$ & 6358 & 15202 & 5512 & 96 \\
\hline $\mathrm{H}(34 \mathrm{~B})$ & 6083 & 14322 & 5882 & 96 \\
\hline
\end{tabular}




\begin{tabular}{lrrrr}
$\mathrm{H}(34 \mathrm{C})$ & 5614 & 14704 & 5109 & 96 \\
$\mathrm{H}(35 \mathrm{~A})$ & 8429 & 10643 & 4228 & 28 \\
$\mathrm{H}(37 \mathrm{~A})$ & 7013 & 10717 & 2602 & 38 \\
$\mathrm{H}(38 \mathrm{~A})$ & 6912 & 11801 & 1670 & 42 \\
$\mathrm{H}(40 \mathrm{~A})$ & 8930 & 12566 & 2592 & 40 \\
$\mathrm{H}(41 \mathrm{~A})$ & 9028 & 11499 & 3533 & 36 \\
$\mathrm{H}(42 \mathrm{~A})$ & 8288 & 13378 & 1528 & 71 \\
$\mathrm{H}(42 \mathrm{~B})$ & 7486 & 13549 & 1436 & 71 \\
$\mathrm{H}(42 \mathrm{C})$ & 7696 & 12699 & 989 & 71 \\
$\mathrm{H}(44 \mathrm{~A})$ & 9154 & 9288 & 4745 & 47 \\
$\mathrm{H}(45 \mathrm{~A})$ & 9854 & 7988 & 4631 & 58 \\
$\mathrm{H}(47 \mathrm{~A})$ & 8752 & 7835 & 2540 & 49 \\
$\mathrm{H}(48 \mathrm{~A})$ & 8063 & 9160 & 2654 & 42 \\
$\mathrm{H}(49 \mathrm{~A})$ & 9711 & 6746 & 2994 & 101 \\
$\mathrm{H}(49 \mathrm{~B})$ & 9852 & 6409 & 3797 & 101 \\
$\mathrm{H}(49 \mathrm{C})$ & 10341 & 7228 & 3628 & 101 \\
$\mathrm{H}(50 \mathrm{~A})$ & 5902 & 9587 & 2918 & 41 \\
$\mathrm{H}(51 \mathrm{~A})$ & 5142 & 9591 & 1753 & 53 \\
$\mathrm{H}(52 \mathrm{~A})$ & 5390 & 8614 & 902 & 48 \\
$\mathrm{H}(53 \mathrm{~A})$ & 6439 & 7718 & 1257 & 44 \\
$\mathrm{H}(54 \mathrm{~A})$ & 7154 & 7731 & 2434 & 37 \\
\hline
\end{tabular}




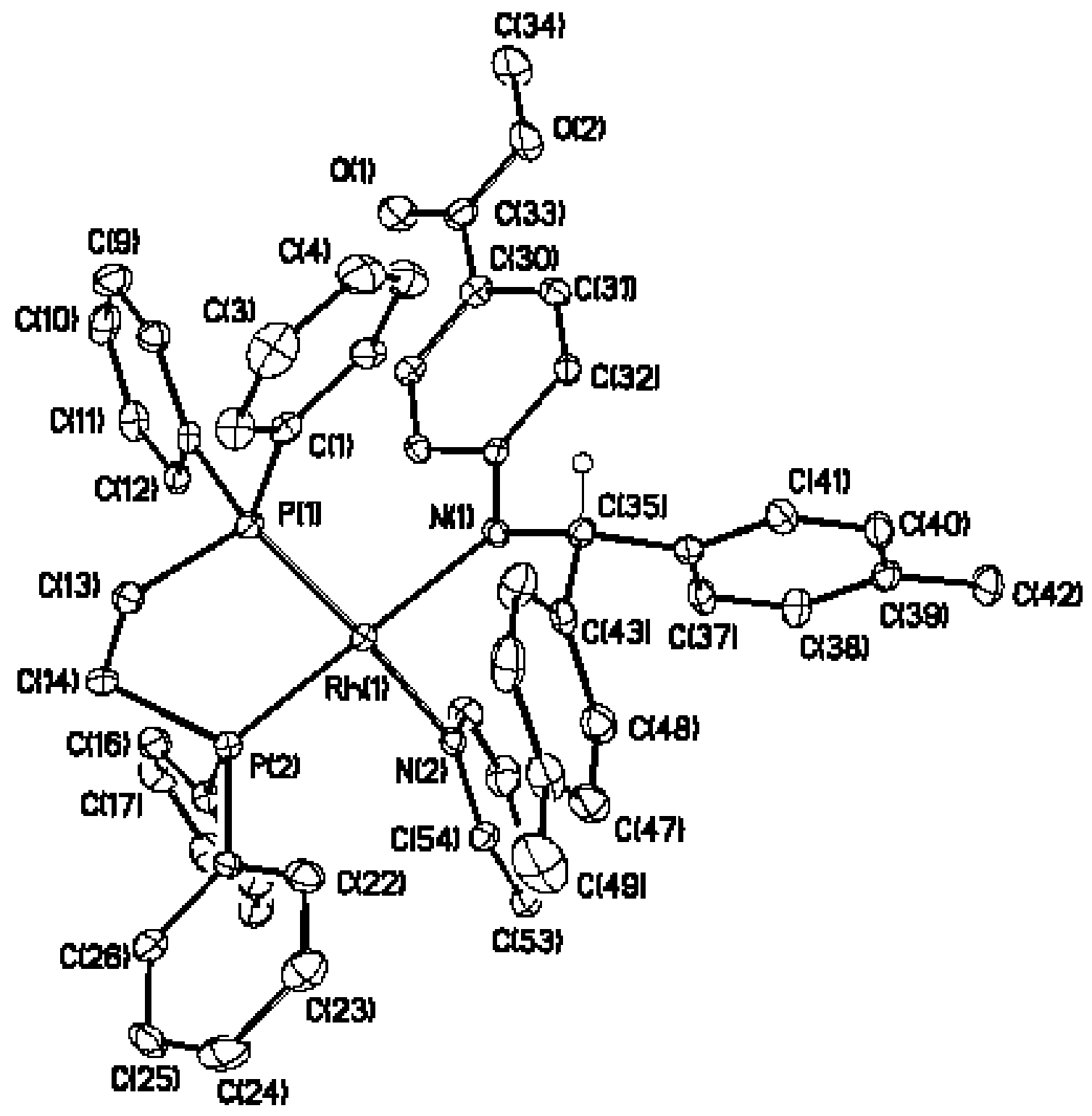

Figure S3. ORTEP diagram of (DPPE) Rh(pyr) $\left(\mathrm{NArCH}(p \text {-tol })_{2}(4)\left(\mathrm{Ar}=\mathrm{C}_{6} \mathrm{H}_{4}-p-\mathrm{CO}_{2} \mathrm{Me}\right)\right.$. 
Experimental Procedure for the X-ray Diffraction of 8 .

\section{$\underline{\text { Data Collection }}$}

A dark red needle crystal of $\mathrm{C}_{48} \mathrm{H}_{44} \mathrm{NOP}_{2} \mathrm{Rh}$ having approximate dimensions of $0.35 \mathrm{x}$ $0.10 \times 0.08 \mathrm{~mm}$ was mounted with epoxy cement on the tip of a fine glass fiber. All measurements were made on a Nonius KappaCCD diffractometer with graphite monochromated Mo-K $\square$ radiation.

Cell constants and an orientation matrix for data collection corresponded to a $C$-centered monoclinic cell with dimensions:

$$
\begin{array}{lll}
\mathrm{a}=14.720(3) \AA & \square=90 \mathrm{o} \\
\mathrm{b}=29.946(6) \AA & \square=98.62(3) \mathrm{o} \\
\mathrm{c}=18.379(4) \AA & \square=90 \mathrm{o} \\
\mathrm{V}=8010(3) \AA 3 &
\end{array}
$$

For $\mathrm{Z}=8$ and F.W. $=815.69$, the calculated density is $1.353 \mathrm{~g} / \mathrm{cm}^{3}$. Based on a statistical analysis of intensity distribution, and the successful solution and refinement of the structure, the space group was determined to be: $C 2 / c(\# 15)$

The data were collected at a temperature of $173(2) \mathrm{K}$ to a maximum $2 \square$ value of $56.64 \mathrm{O}$. Four omega scans consisting of 42, 38, 42, and 24 data frames, respectively, were collected with a frame width of $1.8^{\circ}$ and a detector-to-crystal distance, Dx, of $35.0 \mathrm{~mm}$. Each frame was exposed twice (for the purpose of de-zingering) for a total of 216 seconds. The data frames were processed and scaled using the DENZO software package. ${ }^{1}$

\section{Data Reduction}

A total of 17301 reflections were collected of which 9923 were unique and observed $\left(\mathrm{R}_{\text {int }}\right.$ $=0.0798)$. The linear absorption coefficient, $\square$, for Mo-K $\square$ radiation is $5.43 \mathrm{~cm}^{-1}$ and no absorption correction was applied. The data were corrected for Lorentz and polarization effects.

\section{$\underline{\text { Structure Solution and Refinement }}$}

The structure was solved by direct methods and expanded using Fourier techniques ${ }^{2}$. The non-hydrogen atoms were refined anisotropically and hydrogen atoms were treated as idealized contributions. The final cycle of full-matrix least-squares refinement ${ }^{3}$ on $\mathrm{F}$ was based on 9923 observed reflections (I $>2.00 \square(\mathrm{I})$ ) and 478 variable parameters and converged with unweighted and weighted agreement factors of: 


$$
\begin{gathered}
\mathrm{R}=\mathrm{\square}\|\mathrm{Fo}|-| \mathrm{Fc}\| / \mathrm{\square}|\mathrm{Fo}|=0.0555 \\
\mathrm{R}_{\mathrm{W}}=\left\{\square\left[\mathrm{w}\left(\mathrm{F}_{\mathrm{o}}{ }^{2}-\mathrm{F}_{\mathrm{c}}{ }^{2}\right)^{2}\right] / \square\left[\mathrm{w}\left(\mathrm{F}_{\mathrm{o}}{ }^{2}\right)^{2}\right]\right\}^{1 / 2}=0.0916
\end{gathered}
$$

The maximum and minimum peaks on the final difference Fourier map corresponded to 0.429 and $-0.768 \mathrm{e}^{-} / \AA^{3}$, respectively.

\section{REFERENCES}

(1) Z. Otwinowski and W. Minor, "Processing of X-Ray Diffraction Data Collected in Oscillation Mode," Methods in Enzymology, vol. 276: Macromolecular Crystallography, part A, 307-326, 1997, C.W. Carter, Jr. \& R.M. Sweet, Eds., Academic Press.

(2) Acta Cryst. A46 (1990) 467-473

(3) Least Squares function minimized:

$$
\square w\left(\mathrm{~F}_{\mathrm{o}}{ }^{2}-\mathrm{F}_{\mathrm{c}}{ }^{2}\right)^{2}
$$


Table S11. Crystal data and structure refinement for $\mathbf{8}$.

Empirical formula

Formula weight

Temperature

Wavelength

Crystal system

Space group

Unit cell dimensions

Volume

Z

Density (calculated)

Absorption coefficient

$\mathrm{F}(000)$

Crystal size

Theta range for data collection

Index ranges

Reflections collected

Independent reflections

Completeness to theta $=28.32^{\circ}$

Absorption correction

Max. and min. transmission

Refinement method

Data / restraints / parameters

Goodness-of-fit on $\mathrm{F}^{2}$

Final $\mathrm{R}$ indices [I $>2 \operatorname{sigma}(\mathrm{I})]$

$\mathrm{R}$ indices (all data)

Largest diff. peak and hole
$\mathrm{C}_{48} \mathrm{H}_{44} \mathrm{~N} \mathrm{O} \mathrm{P}_{2} \mathrm{Rh}$

815.69

173(2) K

$0.71073 \AA$

Monoclinic

$\mathrm{C} 2 / \mathrm{c}$

$\mathrm{a}=14.720(3) \AA$

$\square=90^{\circ}$.

$\mathrm{b}=29.946(6) \AA$

$\square=98.62(3)^{\circ}$.

$\mathrm{c}=18.379(4) \AA$

$\square=90^{\circ}$.

8010(3) $\AA^{3}$

8

$1.353 \mathrm{~g} / \mathrm{cm}^{3}$

$5.43 \mathrm{~cm}^{-1}$

3376

$0.35 \times 0.10 \times 0.08 \mathrm{~mm}^{3}$

2.47 to $28.32^{\circ}$.

$-19<=\mathrm{h}<=19,-39<=\mathrm{k}<=39,-24<=1<=24$

17301

$9923[\mathrm{R}(\mathrm{int})=0.0798]$

$99.3 \%$

None

0.9578 and 0.8326

Full-matrix least-squares on $\mathrm{F}^{2}$

9923 / 0 / 478

1.000

$\mathrm{R} 1=0.0555, \mathrm{wR} 2=0.0916$

$\mathrm{R} 1=0.1402, \mathrm{wR} 2=0.1125$

0.429 and -0.768 e. $\AA^{-3}$ 
Table S12. Atomic coordinates ( $\left.\times 10^{4}\right)$ and equivalent isotropic displacement parameters $\left(\AA^{2} \times 10^{3}\right)$ for 8 . $U(e q)$ is defined as one third of the trace of the orthogonalized $U^{i j}$ tensor.

\begin{tabular}{|c|c|c|c|c|}
\hline & $\mathrm{x}$ & $\mathrm{y}$ & $\mathrm{z}$ & $\mathrm{U}(\mathrm{eq})$ \\
\hline $\mathrm{Rh}(1)$ & $6499(1)$ & $1417(1)$ & $4607(1)$ & $29(1)$ \\
\hline $\mathrm{P}(1)$ & $5086(1)$ & $1509(1)$ & $4948(1)$ & $32(1)$ \\
\hline $\mathrm{P}(2)$ & $6447(1)$ & $751(1)$ & $5096(1)$ & $34(1)$ \\
\hline $\mathrm{O}(1)$ & $4427(2)$ & $3546(1)$ & $3928(2)$ & $49(1)$ \\
\hline $\mathrm{N}(1)$ & $6659(2)$ & $2030(1)$ & $4076(1)$ & $28(1)$ \\
\hline $\mathrm{C}(1)$ & $4167(3)$ & $1348(1)$ & $4206(2)$ & $34(1)$ \\
\hline$C(2)$ & $3277(3)$ & $1238(1)$ & $4325(2)$ & $45(1)$ \\
\hline$C(3)$ & 2621(3) & $1089(1)$ & $3750(3)$ & $54(1)$ \\
\hline$C(4)$ & $2848(3)$ & $1055(1)$ & $3047(3)$ & $54(1)$ \\
\hline$C(5)$ & $3704(3)$ & 1173(1) & $2919(2)$ & $52(1)$ \\
\hline$C(6)$ & 4364(3) & $1314(1)$ & $3496(2)$ & $40(1)$ \\
\hline$C(7)$ & $4611(2)$ & $2010(1)$ & $5324(2)$ & $31(1)$ \\
\hline $\mathrm{C}(8)$ & 4933(3) & $2140(1)$ & $6042(2)$ & $43(1)$ \\
\hline$C(9)$ & $4618(3)$ & $2532(1)$ & $6322(2)$ & $51(1)$ \\
\hline$C(10)$ & 4001(3) & $2803(1)$ & $5893(2)$ & $50(1)$ \\
\hline$C(11)$ & $3681(3)$ & $2679(1)$ & $5181(2)$ & $45(1)$ \\
\hline$C(12)$ & $3986(3)$ & $2286(1)$ & $4900(2)$ & $39(1)$ \\
\hline$C(13)$ & 4955(3) & 1081(1) & $5656(2)$ & $39(1)$ \\
\hline$C(14)$ & $5329(3)$ & $643(1)$ & $5405(2)$ & $38(1)$ \\
\hline$C(15)$ & $6528(3)$ & $280(1)$ & $4466(2)$ & $36(1)$ \\
\hline$C(16)$ & $7052(3)$ & $-100(1)$ & $4626(2)$ & $44(1)$ \\
\hline$C(17)$ & 7113(3) & $-418(1)$ & $4084(3)$ & $54(1)$ \\
\hline$C(18)$ & $6662(4)$ & $-357(1)$ & $3387(3)$ & $64(1)$ \\
\hline$C(19)$ & $6139(4)$ & $17(2)$ & $3224(2)$ & $69(2)$ \\
\hline$C(20)$ & $6073(3)$ & $334(1)$ & $3764(2)$ & $55(1)$ \\
\hline$C(21)$ & $7295(3)$ & $627(1)$ & $5913(2)$ & $35(1)$ \\
\hline$C(22)$ & $7256(3)$ & $259(1)$ & $6364(2)$ & $45(1)$ \\
\hline$C(23)$ & $7909(3)$ & $189(2)$ & $6969(2)$ & $56(1)$ \\
\hline$C(24)$ & $8619(3)$ & $487(2)$ & $7140(2)$ & $58(1)$ \\
\hline$C(25)$ & $8675(3)$ & $858(1)$ & $6700(2)$ & $55(1)$ \\
\hline$C(26)$ & 8012(3) & $925(1)$ & $6088(2)$ & $40(1)$ \\
\hline
\end{tabular}




\begin{tabular}{|c|c|c|c|c|}
\hline$C(27)$ & $7299(2)$ & 2033(1) & $3654(2)$ & $28(1)$ \\
\hline$C(28)$ & $7892(2)$ & $1638(1)$ & $3692(2)$ & $29(1)$ \\
\hline$C(29)$ & $7673(2)$ & $1286(1)$ & $4151(2)$ & $31(1)$ \\
\hline$C(30)$ & $8262(3)$ & $917(1)$ & $4181(2)$ & $36(1)$ \\
\hline$C(31)$ & $9013(3)$ & $887(1)$ & $3811(2)$ & $38(1)$ \\
\hline$C(32)$ & $9219(3)$ & $1245(1)$ & $3389(2)$ & $40(1)$ \\
\hline$C(33)$ & $8659(3)$ & 1621(1) & $3327(2)$ & $37(1)$ \\
\hline$C(34)$ & $9611(3)$ & $472(1)$ & $3882(2)$ & $55(1)$ \\
\hline$C(35)$ & $7435(2)$ & 2411(1) & $3152(2)$ & $29(1)$ \\
\hline$C(36)$ & $7362(3)$ & $2328(1)$ & $2400(2)$ & $37(1)$ \\
\hline$C(37)$ & $7500(3)$ & $2668(1)$ & $1919(2)$ & $39(1)$ \\
\hline$C(38)$ & $7729(2)$ & $3095(1)$ & $2170(2)$ & $36(1)$ \\
\hline$C(39)$ & $7800(3)$ & $3175(1)$ & $2922(2)$ & $40(1)$ \\
\hline $\mathrm{C}(40)$ & $7654(3)$ & $2839(1)$ & $3409(2)$ & $37(1)$ \\
\hline $\mathrm{C}(41)$ & $7880(3)$ & $3465(1)$ & $1645(2)$ & $57(1)$ \\
\hline$C(42)$ & $6066(2)$ & $2410(1)$ & $4065(2)$ & $27(1)$ \\
\hline $\mathrm{C}(43)$ & $6157(2)$ & $2705(1)$ & $4650(2)$ & $30(1)$ \\
\hline $\mathrm{C}(44)$ & $5627(3)$ & $3088(1)$ & $4628(2)$ & $35(1)$ \\
\hline$C(45)$ & 4987(3) & $3172(1)$ & $4017(2)$ & $35(1)$ \\
\hline $\mathrm{C}(46)$ & 4863(3) & $2872(1)$ & $3434(2)$ & $37(1)$ \\
\hline $\mathrm{C}(47)$ & $5402(3)$ & 2493(1) & $3464(2)$ & $33(1)$ \\
\hline $\mathrm{C}(48)$ & $4548(3)$ & $3867(1)$ & $4507(2)$ & $61(1)$ \\
\hline
\end{tabular}


Table S13. Bond lengths $[\AA]$ and angles $\left[{ }^{\circ}\right]$ for 8 .

\begin{tabular}{|c|c|c|c|}
\hline $\mathrm{Rh}(1)-\mathrm{C}(29)$ & $2.068(4)$ & $\mathrm{C}(21)-\mathrm{C}(22)$ & $1.388(5)$ \\
\hline $\mathrm{Rh}(1)-\mathrm{N}(1)$ & $2.108(3)$ & $\mathrm{C}(22)-\mathrm{C}(23)$ & $1.372(5)$ \\
\hline $\mathrm{Rh}(1)-\mathrm{P}(2)$ & $2.1939(10)$ & $\mathrm{C}(23)-\mathrm{C}(24)$ & $1.376(6)$ \\
\hline $\mathrm{Rh}(1)-\mathrm{P}(1)$ & $2.2766(11)$ & $\mathrm{C}(24)-\mathrm{C}(25)$ & $1.381(6)$ \\
\hline $\mathrm{P}(1)-\mathrm{C}(7)$ & $1.835(3)$ & $C(25)-C(26)$ & $1.389(5)$ \\
\hline $\mathrm{P}(1)-\mathrm{C}(1)$ & $1.834(4)$ & $\mathrm{C}(27)-\mathrm{C}(28)$ & $1.466(5)$ \\
\hline $\mathrm{P}(1)-\mathrm{C}(13)$ & $1.857(3)$ & $\mathrm{C}(27)-\mathrm{C}(35)$ & $1.493(4)$ \\
\hline $\mathrm{P}(2)-\mathrm{C}(21)$ & $1.840(4)$ & $\mathrm{C}(28)-\mathrm{C}(33)$ & $1.398(5)$ \\
\hline $\mathrm{P}(2)-\mathrm{C}(15)$ & $1.840(4)$ & $C(28)-C(29)$ & $1.417(5)$ \\
\hline $\mathrm{P}(2)-\mathrm{C}(14)$ & $1.849(4)$ & $\mathrm{C}(29)-\mathrm{C}(30)$ & $1.401(5)$ \\
\hline $\mathrm{O}(1)-\mathrm{C}(45)$ & $1.383(4)$ & $\mathrm{C}(30)-\mathrm{C}(31)$ & $1.386(5)$ \\
\hline $\mathrm{O}(1)-\mathrm{C}(48)$ & $1.424(4)$ & $\mathrm{C}(31)-\mathrm{C}(32)$ & $1.381(5)$ \\
\hline $\mathrm{N}(1)-\mathrm{C}(27)$ & $1.306(4)$ & $\mathrm{C}(31)-\mathrm{C}(34)$ & $1.516(5)$ \\
\hline $\mathrm{N}(1)-\mathrm{C}(42)$ & $1.434(4)$ & $\mathrm{C}(32)-\mathrm{C}(33)$ & $1.391(5)$ \\
\hline$C(1)-C(6)$ & $1.384(5)$ & $\mathrm{C}(35)-\mathrm{C}(40)$ & $1.386(4)$ \\
\hline $\mathrm{C}(1)-\mathrm{C}(2)$ & $1.399(5)$ & $\mathrm{C}(35)-\mathrm{C}(36)$ & $1.392(5)$ \\
\hline $\mathrm{C}(2)-\mathrm{C}(3)$ & $1.394(5)$ & $C(36)-C(37)$ & $1.382(5)$ \\
\hline$C(3)-C(4)$ & $1.385(6)$ & $\mathrm{C}(37)-\mathrm{C}(38)$ & $1.385(5)$ \\
\hline$C(4)-C(5)$ & $1.362(6)$ & $\mathrm{C}(38)-\mathrm{C}(39)$ & $1.392(5)$ \\
\hline$C(5)-C(6)$ & $1.392(5)$ & $\mathrm{C}(38)-\mathrm{C}(41)$ & $1.506(5)$ \\
\hline$C(7)-C(12)$ & $1.386(5)$ & $\mathrm{C}(39)-\mathrm{C}(40)$ & $1.384(5)$ \\
\hline$C(7)-C(8)$ & $1.389(5)$ & $\mathrm{C}(42)-\mathrm{C}(43)$ & $1.382(4)$ \\
\hline $\mathrm{C}(8)-\mathrm{C}(9)$ & $1.390(5)$ & $\mathrm{C}(42)-\mathrm{C}(47)$ & $1.383(5)$ \\
\hline$C(9)-C(10)$ & $1.374(6)$ & $\mathrm{C}(43)-\mathrm{C}(44)$ & $1.384(5)$ \\
\hline$C(10)-C(11)$ & $1.373(5)$ & $\mathrm{C}(44)-\mathrm{C}(45)$ & $1.377(5)$ \\
\hline$C(11)-C(12)$ & $1.385(5)$ & $\mathrm{C}(45)-\mathrm{C}(46)$ & $1.391(5)$ \\
\hline$C(13)-C(14)$ & $1.520(5)$ & $\mathrm{C}(46)-\mathrm{C}(47)$ & $1.381(5)$ \\
\hline$C(15)-C(20)$ & $1.371(5)$ & & \\
\hline$C(15)-C(16)$ & $1.380(5)$ & $\mathrm{C}(29)-\mathrm{Rh}(1)-\mathrm{N}(1)$ & $79.57(12)$ \\
\hline$C(16)-C(17)$ & $1.391(5)$ & $\mathrm{C}(29)-\mathrm{Rh}(1)-\mathrm{P}(2)$ & $94.36(10)$ \\
\hline$C(17)-C(18)$ & $1.363(6)$ & $\mathrm{N}(1)-\mathrm{Rh}(1)-\mathrm{P}(2)$ & $173.84(8)$ \\
\hline$C(18)-C(19)$ & $1.367(6)$ & $\mathrm{C}(29)-\mathrm{Rh}(1)-\mathrm{P}(1)$ & $170.98(10)$ \\
\hline$C(19)-C(20)$ & $1.387(5)$ & $\mathrm{N}(1)-\mathrm{Rh}(1)-\mathrm{P}(1)$ & $101.13(8)$ \\
\hline$C(21)-C(26)$ & $1.381(5)$ & $\mathrm{P}(2)-\mathrm{Rh}(1)-\mathrm{P}(1)$ & $84.70(4)$ \\
\hline
\end{tabular}




\begin{tabular}{|c|c|c|c|}
\hline $\mathrm{C}(7)-\mathrm{P}(1)-\mathrm{C}(1)$ & $102.37(16)$ & $\mathrm{C}(16)-\mathrm{C}(15)-\mathrm{P}(2)$ & $125.7(3)$ \\
\hline$C(7)-P(1)-C(13)$ & $102.42(16)$ & $\mathrm{C}(15)-\mathrm{C}(16)-\mathrm{C}(17)$ & $120.3(4)$ \\
\hline $\mathrm{C}(1)-\mathrm{P}(1)-\mathrm{C}(13)$ & $101.41(17)$ & $\mathrm{C}(18)-\mathrm{C}(17)-\mathrm{C}(16)$ & $120.5(4)$ \\
\hline $\mathrm{C}(7)-\mathrm{P}(1)-\mathrm{Rh}(1)$ & $128.11(12)$ & $\mathrm{C}(17)-\mathrm{C}(18)-\mathrm{C}(19)$ & $119.6(4)$ \\
\hline $\mathrm{C}(1)-\mathrm{P}(1)-\mathrm{Rh}(1)$ & $111.51(12)$ & $C(18)-C(19)-C(20)$ & $120.1(4)$ \\
\hline $\mathrm{C}(13)-\mathrm{P}(1)-\mathrm{Rh}(1)$ & $107.75(13)$ & $C(15)-C(20)-C(19)$ & $121.1(4)$ \\
\hline $\mathrm{C}(21)-\mathrm{P}(2)-\mathrm{C}(15)$ & $105.03(17)$ & $\mathrm{C}(26)-\mathrm{C}(21)-\mathrm{C}(22)$ & $118.2(4)$ \\
\hline $\mathrm{C}(21)-\mathrm{P}(2)-\mathrm{C}(14)$ & $103.91(17)$ & $\mathrm{C}(26)-\mathrm{C}(21)-\mathrm{P}(2)$ & $117.5(3)$ \\
\hline $\mathrm{C}(15)-\mathrm{P}(2)-\mathrm{C}(14)$ & 101.71(16) & $\mathrm{C}(22)-\mathrm{C}(21)-\mathrm{P}(2)$ & $124.3(3)$ \\
\hline $\mathrm{C}(21)-\mathrm{P}(2)-\mathrm{Rh}(1)$ & $117.16(12)$ & $\mathrm{C}(23)-\mathrm{C}(22)-\mathrm{C}(21)$ & $121.3(4)$ \\
\hline $\mathrm{C}(15)-\mathrm{P}(2)-\mathrm{Rh}(1)$ & $115.47(12)$ & $\mathrm{C}(22)-\mathrm{C}(23)-\mathrm{C}(24)$ & $120.1(4)$ \\
\hline $\mathrm{C}(14)-\mathrm{P}(2)-\mathrm{Rh}(1)$ & $111.84(12)$ & $\mathrm{C}(23)-\mathrm{C}(24)-\mathrm{C}(25)$ & $119.9(4)$ \\
\hline $\mathrm{C}(45)-\mathrm{O}(1)-\mathrm{C}(48)$ & $116.8(3)$ & $\mathrm{C}(24)-\mathrm{C}(25)-\mathrm{C}(26)$ & $119.6(4)$ \\
\hline $\mathrm{C}(27)-\mathrm{N}(1)-\mathrm{C}(42)$ & $118.5(3)$ & $\mathrm{C}(21)-\mathrm{C}(26)-\mathrm{C}(25)$ & $121.0(4)$ \\
\hline $\mathrm{C}(27)-\mathrm{N}(1)-\mathrm{Rh}(1)$ & $115.0(2)$ & $\mathrm{N}(1)-\mathrm{C}(27)-\mathrm{C}(28)$ & $116.3(3)$ \\
\hline $\mathrm{C}(42)-\mathrm{N}(1)-\mathrm{Rh}(1)$ & $126.0(2)$ & $\mathrm{N}(1)-\mathrm{C}(27)-\mathrm{C}(35)$ & $123.3(3)$ \\
\hline $\mathrm{C}(6)-\mathrm{C}(1)-\mathrm{C}(2)$ & $117.6(4)$ & $\mathrm{C}(28)-\mathrm{C}(27)-\mathrm{C}(35)$ & $120.4(3)$ \\
\hline $\mathrm{C}(6)-\mathrm{C}(1)-\mathrm{P}(1)$ & $119.0(3)$ & $\mathrm{C}(33)-\mathrm{C}(28)-\mathrm{C}(29)$ & $122.1(3)$ \\
\hline $\mathrm{C}(2)-\mathrm{C}(1)-\mathrm{P}(1)$ & $123.4(3)$ & $\mathrm{C}(33)-\mathrm{C}(28)-\mathrm{C}(27)$ & $121.7(3)$ \\
\hline $\mathrm{C}(3)-\mathrm{C}(2)-\mathrm{C}(1)$ & 121.1(4) & $\mathrm{C}(29)-\mathrm{C}(28)-\mathrm{C}(27)$ & $116.2(3)$ \\
\hline$C(2)-C(3)-C(4)$ & $119.6(4)$ & $\mathrm{C}(30)-\mathrm{C}(29)-\mathrm{C}(28)$ & $114.5(3)$ \\
\hline $\mathrm{C}(5)-\mathrm{C}(4)-\mathrm{C}(3)$ & $120.0(4)$ & $\mathrm{C}(30)-\mathrm{C}(29)-\mathrm{Rh}(1)$ & $133.1(3)$ \\
\hline$C(4)-C(5)-C(6)$ & $120.4(4)$ & $\mathrm{C}(28)-\mathrm{C}(29)-\mathrm{Rh}(1)$ & $112.3(2)$ \\
\hline$C(1)-C(6)-C(5)$ & $121.3(4)$ & $\mathrm{C}(31)-\mathrm{C}(30)-\mathrm{C}(29)$ & $124.6(3)$ \\
\hline $\mathrm{C}(12)-\mathrm{C}(7)-\mathrm{C}(8)$ & $118.0(3)$ & $\mathrm{C}(32)-\mathrm{C}(31)-\mathrm{C}(30)$ & $118.9(3)$ \\
\hline$C(12)-C(7)-P(1)$ & $122.3(3)$ & $\mathrm{C}(32)-\mathrm{C}(31)-\mathrm{C}(34)$ & $120.7(3)$ \\
\hline $\mathrm{C}(8)-\mathrm{C}(7)-\mathrm{P}(1)$ & $119.5(3)$ & $\mathrm{C}(30)-\mathrm{C}(31)-\mathrm{C}(34)$ & $120.4(3)$ \\
\hline $\mathrm{C}(9)-\mathrm{C}(8)-\mathrm{C}(7)$ & $120.2(4)$ & $\mathrm{C}(31)-\mathrm{C}(32)-\mathrm{C}(33)$ & $119.8(4)$ \\
\hline$C(10)-C(9)-C(8)$ & $121.0(4)$ & $\mathrm{C}(32)-\mathrm{C}(33)-\mathrm{C}(28)$ & $120.1(3)$ \\
\hline $\mathrm{C}(11)-\mathrm{C}(10)-\mathrm{C}(9)$ & 119.3(4) & $\mathrm{C}(40)-\mathrm{C}(35)-\mathrm{C}(36)$ & $118.9(3)$ \\
\hline$C(10)-C(11)-C(12)$ & $120.1(4)$ & $\mathrm{C}(40)-\mathrm{C}(35)-\mathrm{C}(27)$ & $122.4(3)$ \\
\hline$C(11)-C(12)-C(7)$ & $121.4(4)$ & $\mathrm{C}(36)-\mathrm{C}(35)-\mathrm{C}(27)$ & $118.7(3)$ \\
\hline $\mathrm{C}(14)-\mathrm{C}(13)-\mathrm{P}(1)$ & $107.6(2)$ & $\mathrm{C}(37)-\mathrm{C}(36)-\mathrm{C}(35)$ & $120.4(3)$ \\
\hline $\mathrm{C}(13)-\mathrm{C}(14)-\mathrm{P}(2)$ & $108.7(2)$ & $\mathrm{C}(36)-\mathrm{C}(37)-\mathrm{C}(38)$ & $121.3(3)$ \\
\hline$C(20)-C(15)-C(16)$ & $118.4(4)$ & $\mathrm{C}(37)-\mathrm{C}(38)-\mathrm{C}(39)$ & $117.9(3)$ \\
\hline $\mathrm{C}(20)-\mathrm{C}(15)-\mathrm{P}(2)$ & $115.7(3)$ & $\mathrm{C}(37)-\mathrm{C}(38)-\mathrm{C}(41)$ & $121.2(3)$ \\
\hline
\end{tabular}




$\begin{array}{ll}\mathrm{C}(39)-\mathrm{C}(38)-\mathrm{C}(41) & 120.9(4) \\ \mathrm{C}(40)-\mathrm{C}(39)-\mathrm{C}(38) & 121.4(3) \\ \mathrm{C}(39)-\mathrm{C}(40)-\mathrm{C}(35) & 120.2(3) \\ \mathrm{C}(43)-\mathrm{C}(42)-\mathrm{C}(47) & 118.8(3) \\ \mathrm{C}(43)-\mathrm{C}(42)-\mathrm{N}(1) & 120.6(3) \\ \mathrm{C}(47)-\mathrm{C}(42)-\mathrm{N}(1) & 120.6(3) \\ \mathrm{C}(42)-\mathrm{C}(43)-\mathrm{C}(44) & 121.2(3) \\ \mathrm{C}(45)-\mathrm{C}(44)-\mathrm{C}(43) & 119.3(3) \\ \mathrm{C}(44)-\mathrm{C}(45)-\mathrm{O}(1) & 124.6(3) \\ \mathrm{C}(44)-\mathrm{C}(45)-\mathrm{C}(46) & 120.3(3) \\ \mathrm{O}(1)-\mathrm{C}(45)-\mathrm{C}(46) & 115.1(3) \\ \mathrm{C}(47)-\mathrm{C}(46)-\mathrm{C}(45) & 119.5(3) \\ \mathrm{C}(46)-\mathrm{C}(47)-\mathrm{C}(42) & 120.8(3)\end{array}$


Table S14. Anisotropic displacement parameters $\left(\AA^{2} \times 10^{3}\right)$ for 8 . The anisotropic displacement factor exponent takes the form: $-2 \square^{2}\left[h^{2} a^{* 2} U^{11}+\ldots+2 h k a^{*} b^{*} U^{12}\right]$

\begin{tabular}{|c|c|c|c|c|c|c|}
\hline & $\mathrm{U}^{11}$ & $\mathrm{U}^{22}$ & $\mathrm{U}^{33}$ & $\mathrm{U}^{23}$ & $\mathrm{U}^{13}$ & $\mathrm{U}^{12}$ \\
\hline $\mathrm{Rh}(1)$ & $29(1)$ & $26(1)$ & $35(1)$ & $4(1)$ & 11(1) & $0(1)$ \\
\hline $\mathrm{P}(1)$ & $29(1)$ & $32(1)$ & $38(1)$ & $2(1)$ & 11(1) & $1(1)$ \\
\hline $\mathrm{P}(2)$ & $34(1)$ & $27(1)$ & $42(1)$ & $6(1)$ & $12(1)$ & $1(1)$ \\
\hline $\mathrm{O}(1)$ & $40(2)$ & $41(2)$ & $63(2)$ & $-6(1)$ & $-1(1)$ & $15(1)$ \\
\hline $\mathrm{N}(1)$ & $28(2)$ & $24(2)$ & $32(2)$ & $2(1)$ & $6(1)$ & $-5(1)$ \\
\hline $\mathrm{C}(1)$ & $36(2)$ & $27(2)$ & $39(2)$ & $-1(2)$ & $8(2)$ & $3(2)$ \\
\hline$C(2)$ & $39(3)$ & $46(2)$ & $51(3)$ & $3(2)$ & $7(2)$ & $-7(2)$ \\
\hline$C(3)$ & $38(3)$ & $44(3)$ & $75(3)$ & $9(2)$ & $-1(2)$ & $-14(2)$ \\
\hline$C(4)$ & $59(3)$ & $33(2)$ & $63(3)$ & $-1(2)$ & $-13(3)$ & $-4(2)$ \\
\hline$C(5)$ & $65(3)$ & $43(3)$ & $44(3)$ & $-6(2)$ & $-2(2)$ & $5(2)$ \\
\hline$C(6)$ & $43(3)$ & $38(2)$ & $41(2)$ & $-2(2)$ & $8(2)$ & $3(2)$ \\
\hline$C(7)$ & $25(2)$ & $32(2)$ & $40(2)$ & $0(2)$ & $13(2)$ & $1(2)$ \\
\hline$C(8)$ & $39(3)$ & $49(3)$ & $42(2)$ & $0(2)$ & $7(2)$ & $0(2)$ \\
\hline$C(9)$ & $60(3)$ & $50(3)$ & $45(3)$ & $-18(2)$ & $13(2)$ & $-14(2)$ \\
\hline$C(10)$ & $51(3)$ & $35(2)$ & $68(3)$ & $-15(2)$ & $25(2)$ & $-8(2)$ \\
\hline $\mathrm{C}(11)$ & $34(3)$ & $35(2)$ & $65(3)$ & $-6(2)$ & $11(2)$ & $1(2)$ \\
\hline $\mathrm{C}(12)$ & $38(3)$ & $34(2)$ & $45(2)$ & $-1(2)$ & $12(2)$ & $1(2)$ \\
\hline $\mathrm{C}(13)$ & $37(3)$ & $40(2)$ & $42(2)$ & $4(2)$ & $14(2)$ & $-2(2)$ \\
\hline$C(14)$ & $35(2)$ & $35(2)$ & $47(2)$ & $6(2)$ & $13(2)$ & $-3(2)$ \\
\hline$C(15)$ & $36(2)$ & $30(2)$ & $45(2)$ & $6(2)$ & $13(2)$ & $-9(2)$ \\
\hline$C(16)$ & $43(3)$ & $32(2)$ & $59(3)$ & $2(2)$ & $13(2)$ & $-1(2)$ \\
\hline $\mathrm{C}(17)$ & $61(3)$ & $32(2)$ & $75(3)$ & $-3(2)$ & $28(3)$ & $0(2)$ \\
\hline $\mathrm{C}(18)$ & $105(4)$ & $35(3)$ & $56(3)$ & $-7(2)$ & $29(3)$ & $-5(3)$ \\
\hline$C(19)$ & $114(5)$ & $49(3)$ & $46(3)$ & $-4(2)$ & $14(3)$ & $4(3)$ \\
\hline$C(20)$ & 79(4) & $39(2)$ & $47(3)$ & $5(2)$ & $14(2)$ & $9(2)$ \\
\hline $\mathrm{C}(21)$ & $37(2)$ & $33(2)$ & $37(2)$ & $3(2)$ & $12(2)$ & $7(2)$ \\
\hline $\mathrm{C}(22)$ & $48(3)$ & $40(2)$ & $46(2)$ & $8(2)$ & $10(2)$ & $-5(2)$ \\
\hline $\mathrm{C}(23)$ & $57(3)$ & $59(3)$ & $53(3)$ & $25(2)$ & $10(2)$ & $7(3)$ \\
\hline $\mathrm{C}(24)$ & $50(3)$ & $71(3)$ & $49(3)$ & $15(2)$ & $-2(2)$ & $4(3)$ \\
\hline $\mathrm{C}(25)$ & $48(3)$ & $55(3)$ & $61(3)$ & $1(2)$ & $0(2)$ & $-4(2)$ \\
\hline$C(26)$ & 41(3) & $40(2)$ & $41(2)$ & $3(2)$ & $10(2)$ & $1(2)$ \\
\hline
\end{tabular}




\begin{tabular}{lllllll}
$\mathrm{C}(27)$ & $26(2)$ & $27(2)$ & $30(2)$ & $-1(2)$ & $4(2)$ & $-5(2)$ \\
$\mathrm{C}(28)$ & $24(2)$ & $31(2)$ & $30(2)$ & $-2(2)$ & $5(2)$ & $-5(2)$ \\
$\mathrm{C}(29)$ & $30(2)$ & $26(2)$ & $36(2)$ & $-2(2)$ & $10(2)$ & $0(2)$ \\
$\mathrm{C}(30)$ & $35(2)$ & $25(2)$ & $49(2)$ & $-3(2)$ & $10(2)$ & $-4(2)$ \\
$\mathrm{C}(31)$ & $32(2)$ & $33(2)$ & $48(2)$ & $-7(2)$ & $9(2)$ & $1(2)$ \\
$\mathrm{C}(32)$ & $33(2)$ & $50(2)$ & $40(2)$ & $-3(2)$ & $16(2)$ & $4(2)$ \\
$\mathrm{C}(33)$ & $33(2)$ & $37(2)$ & $44(2)$ & $3(2)$ & $9(2)$ & $0(2)$ \\
$\mathrm{C}(34)$ & $53(3)$ & $47(3)$ & $66(3)$ & $-5(2)$ & $16(2)$ & $14(2)$ \\
$\mathrm{C}(35)$ & $24(2)$ & $29(2)$ & $37(2)$ & $2(2)$ & $10(2)$ & $2(2)$ \\
$\mathrm{C}(36)$ & $38(2)$ & $35(2)$ & $38(2)$ & $3(2)$ & $11(2)$ & $0(2)$ \\
$\mathrm{C}(37)$ & $32(2)$ & $49(2)$ & $37(2)$ & $9(2)$ & $10(2)$ & $-2(2)$ \\
$\mathrm{C}(38)$ & $26(2)$ & $37(2)$ & $46(2)$ & $16(2)$ & $12(2)$ & $-1(2)$ \\
$\mathrm{C}(39)$ & $40(3)$ & $27(2)$ & $54(3)$ & $5(2)$ & $12(2)$ & $0(2)$ \\
$\mathrm{C}(40)$ & $38(2)$ & $35(2)$ & $41(2)$ & $1(2)$ & $13(2)$ & $-3(2)$ \\
$\mathrm{C}(41)$ & $59(3)$ & $49(3)$ & $65(3)$ & $27(2)$ & $17(2)$ & $-6(2)$ \\
$\mathrm{C}(42)$ & $27(2)$ & $28(2)$ & $28(2)$ & $3(2)$ & $6(2)$ & $0(2)$ \\
$\mathrm{C}(43)$ & $26(2)$ & $33(2)$ & $31(2)$ & $1(2)$ & $3(2)$ & $-1(2)$ \\
$\mathrm{C}(44)$ & $36(2)$ & $31(2)$ & $38(2)$ & $-9(2)$ & $4(2)$ & $0(2)$ \\
$\mathrm{C}(45)$ & $29(2)$ & $27(2)$ & $51(2)$ & $0(2)$ & $8(2)$ & $1(2)$ \\
$\mathrm{C}(46)$ & $34(2)$ & $42(2)$ & $33(2)$ & $4(2)$ & $0(2)$ & $4(2)$ \\
$\mathrm{C}(47)$ & $36(2)$ & $37(2)$ & $27(2)$ & $-5(2)$ & $5(2)$ & $0(2)$ \\
$\mathrm{C}(48)$ & $56(3)$ & $40(2)$ & $86(3)$ & $-19(2)$ & $4(3)$ & $14(2)$ \\
& & & & & & \\
\hline
\end{tabular}


Table S15. Hydrogen coordinates ( $\left.\mathrm{x} 10^{4}\right)$ and isotropic displacement parameters $\left(\AA^{2} \times 10^{3}\right)$ for 8 .

\begin{tabular}{|c|c|c|c|c|}
\hline & $\mathrm{x}$ & $\mathrm{y}$ & $\mathrm{z}$ & $\mathrm{U}(\mathrm{eq})$ \\
\hline $\mathrm{H}(2 \mathrm{~A})$ & 3117 & 1265 & 4805 & 54 \\
\hline $\mathrm{H}(3 \mathrm{~A})$ & 2023 & 1010 & 3839 & 64 \\
\hline $\mathrm{H}(4 \mathrm{~A})$ & 2407 & 950 & 2654 & 64 \\
\hline $\mathrm{H}(5 \mathrm{~A})$ & 3851 & 1159 & 2434 & 62 \\
\hline $\mathrm{H}(6 \mathrm{~A})$ & 4962 & 1388 & 3399 & 49 \\
\hline $\mathrm{H}(8 \mathrm{~A})$ & 5370 & 1960 & 6342 & 52 \\
\hline $\mathrm{H}(9 \mathrm{~A})$ & 4832 & 2614 & 6817 & 62 \\
\hline $\mathrm{H}(10 \mathrm{~A})$ & 3797 & 3073 & 6087 & 60 \\
\hline $\mathrm{H}(11 \mathrm{~A})$ & 3251 & 2862 & 4881 & 53 \\
\hline $\mathrm{H}(12 \mathrm{~A})$ & 3761 & 2204 & 4407 & 46 \\
\hline $\mathrm{H}(13 \mathrm{~A})$ & 5301 & 1171 & 6137 & 47 \\
\hline $\mathrm{H}(13 \mathrm{~B})$ & 4299 & 1047 & 5710 & 47 \\
\hline $\mathrm{H}(14 \mathrm{~A})$ & 4892 & 515 & 4996 & 46 \\
\hline $\mathrm{H}(14 \mathrm{~B})$ & 5408 & 426 & 5817 & 46 \\
\hline $\mathrm{H}(16 \mathrm{~A})$ & 7373 & -144 & 5109 & 53 \\
\hline $\mathrm{H}(17 \mathrm{~A})$ & 7472 & -679 & 4200 & 65 \\
\hline $\mathrm{H}(18 \mathrm{~A})$ & 6711 & -573 & 3017 & 76 \\
\hline $\mathrm{H}(19 \mathrm{~A})$ & 5820 & 60 & 2740 & 83 \\
\hline $\mathrm{H}(20 \mathrm{~A})$ & 5706 & 592 & 3646 & 66 \\
\hline $\mathrm{H}(22 \mathrm{~A})$ & 6768 & 50 & 6252 & 54 \\
\hline $\mathrm{H}(23 \mathrm{~A})$ & 7870 & -66 & 7271 & 67 \\
\hline $\mathrm{H}(24 \mathrm{~A})$ & 9070 & 439 & 7559 & 69 \\
\hline $\mathrm{H}(25 \mathrm{~A})$ & 9164 & 1065 & 6816 & 66 \\
\hline $\mathrm{H}(26 \mathrm{~A})$ & 8053 & 1179 & 5785 & 48 \\
\hline $\mathrm{H}(30 \mathrm{~A})$ & 8139 & 671 & 4476 & 43 \\
\hline $\mathrm{H}(32 \mathrm{~A})$ & 9742 & 1233 & 3143 & 48 \\
\hline $\mathrm{H}(33 \mathrm{~A})$ & 8798 & 1867 & 3036 & 45 \\
\hline $\mathrm{H}(34 \mathrm{~A})$ & 10103 & 507 & 3582 & 82 \\
\hline $\mathrm{H}(34 \mathrm{~B})$ & 9235 & 212 & 3711 & 82 \\
\hline $\mathrm{H}(34 \mathrm{C})$ & 9879 & 430 & 4398 & 82 \\
\hline
\end{tabular}




\begin{tabular}{lllll}
$\mathrm{H}(36 \mathrm{~A})$ & 7217 & 2036 & 2217 & 44 \\
$\mathrm{H}(37 \mathrm{~A})$ & 7436 & 2607 & 1407 & 47 \\
$\mathrm{H}(39 \mathrm{~A})$ & 7951 & 3466 & 3106 & 48 \\
$\mathrm{H}(40 \mathrm{~A})$ & 7705 & 2901 & 3920 & 44 \\
$\mathrm{H}(41 \mathrm{~A})$ & 8031 & 3741 & 1921 & 85 \\
$\mathrm{H}(41 \mathrm{~B})$ & 7319 & 3509 & 1291 & 85 \\
$\mathrm{H}(41 \mathrm{C})$ & 8388 & 3384 & 1381 & 85 \\
$\mathrm{H}(43 \mathrm{~A})$ & 6592 & 2644 & 5075 & 36 \\
$\mathrm{H}(44 \mathrm{~A})$ & 5705 & 3291 & 5031 & 42 \\
$\mathrm{H}(46 \mathrm{~A})$ & 4412 & 2927 & 3017 & 44 \\
$\mathrm{H}(47 \mathrm{~A})$ & 5315 & 2286 & 3068 & 40 \\
$\mathrm{H}(48 \mathrm{~A})$ & 4117 & 4114 & 4383 & 92 \\
$\mathrm{H}(48 \mathrm{~B})$ & 5179 & 3980 & 4570 & 92 \\
$\mathrm{H}(48 \mathrm{C})$ & 4430 & 3726 & 4965 & 92 \\
& & & & \\
\hline
\end{tabular}




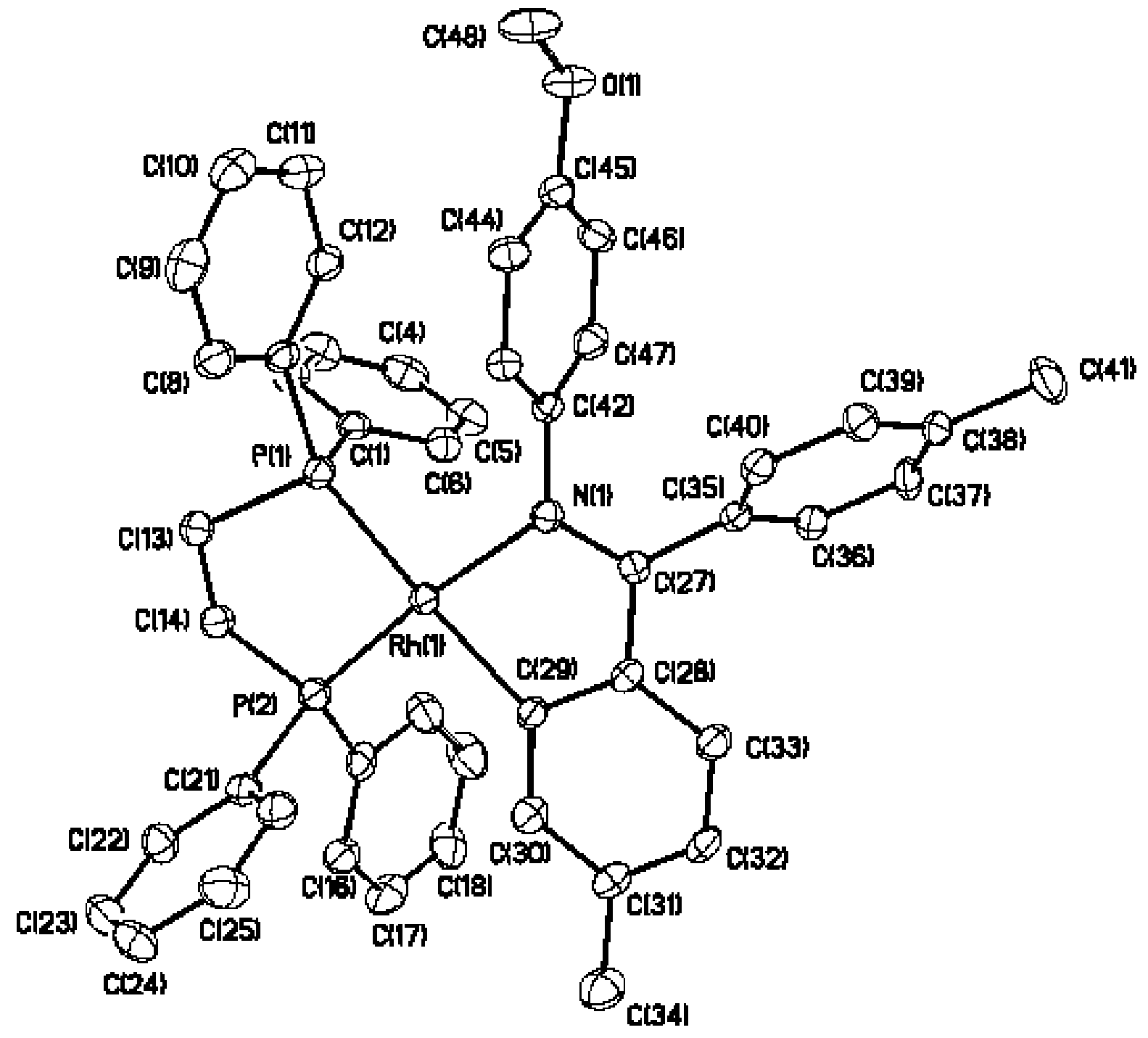

Figure S4. ORTEP diagram of 8 . 


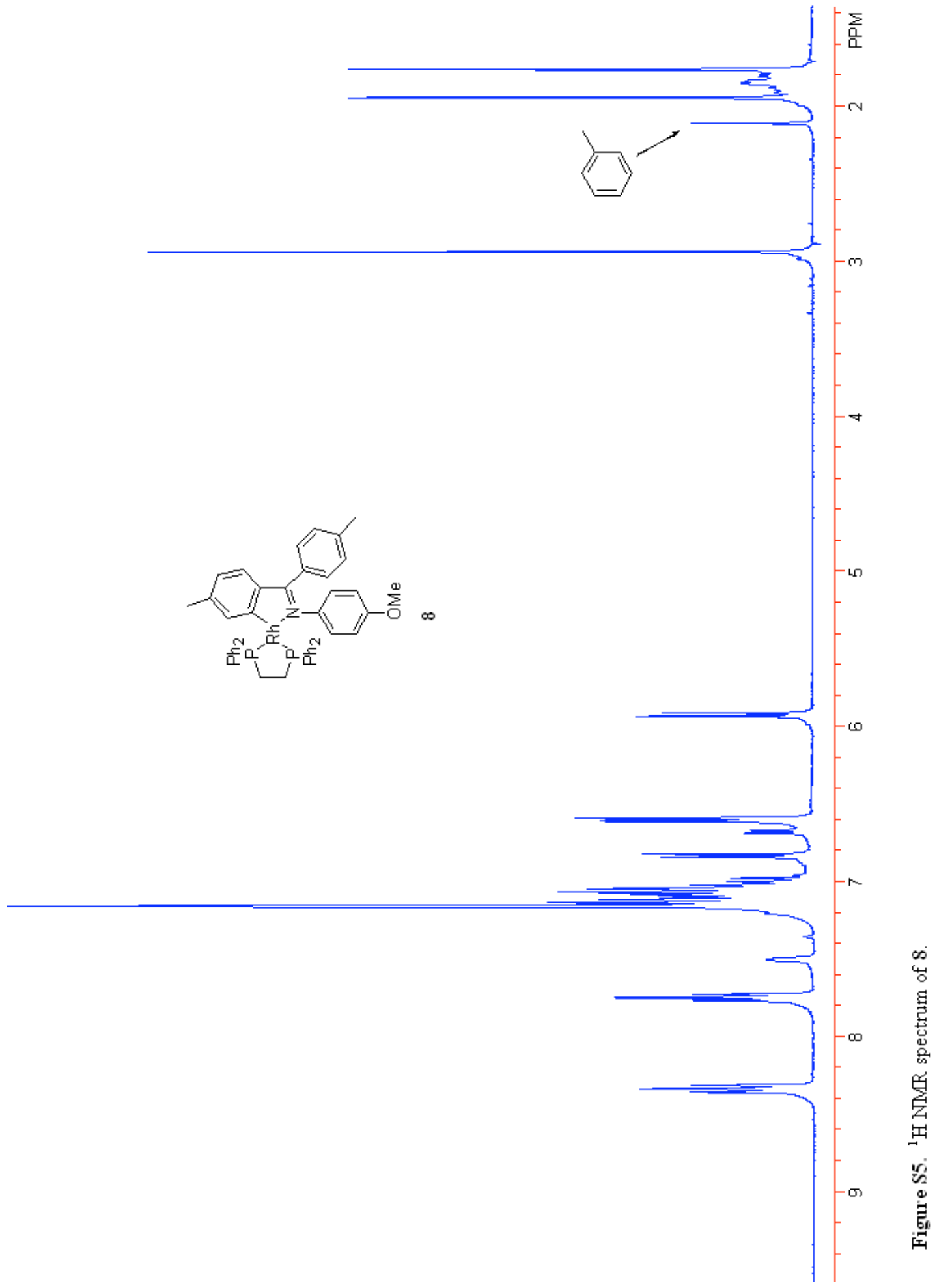



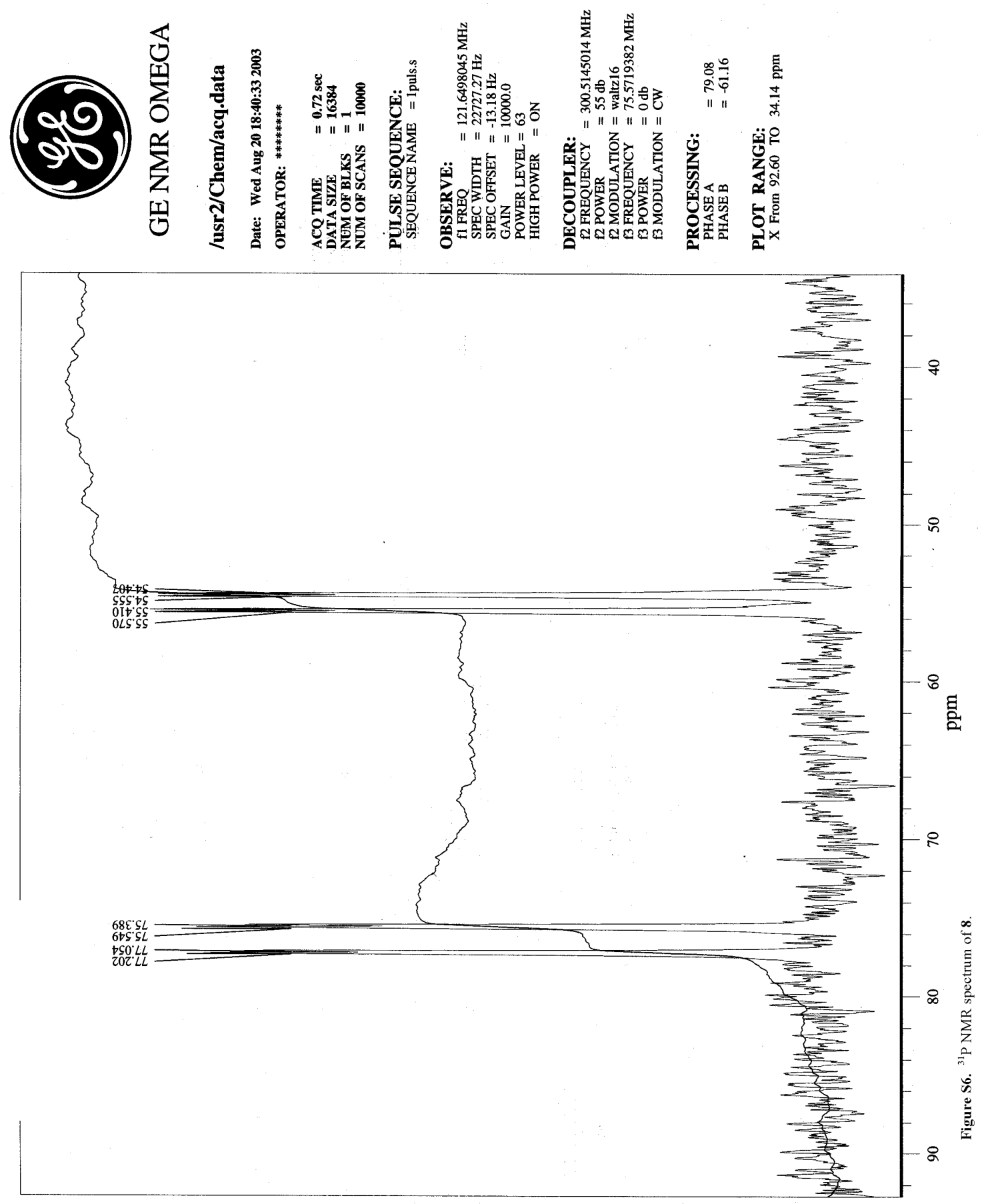